\title{
Circulating miR-181 is a prognostic biomarker for amyotrophic lateral sclerosis
}

Eran Hornstein ( $\nabla$ eran.hornstein@weizmann.ac.il)

Weizmann Institute of Science

\section{Iddo Magen}

Weizmann Institute of Science

\section{Anna Coenen-Stass}

AstraZeneca (United Kingdom)

\section{Nancy Yacovzada}

Weizmann Institute of Science https://orcid.org/0000-0002-5152-8775

Julian Grosskreutz

Jena University Hospital

Ching-Hua Lu

China Medical University Hospital

\section{Linda Greensmith}

UCL

\section{Andrea Malaspina}

Queen Mary University of London

\section{Pietro Fratta}

Department of Neuromuscular Diseases, UCL Queen Square Institute of Neurology https://orcid.org/0000-0002-8762-8188

\section{Eran Yanowski}

Weizmann Institute of Science

\section{Article}

Keywords: Amyotrophic lateral sclerosis (ALS), miR-181, biomarkers

Posted Date: June 12th, 2021

DOl: https://doi.org/10.21203/rs.3.rs-554057/v1

License: (c) (i) This work is licensed under a Creative Commons Attribution 4.0 International License. Read Full License 
Version of Record: A version of this preprint was published at Nature Neuroscience on October 28th, 2021. See the published version at https://doi.org/10.1038/s41593-021-00936-z. 
Circulating miR-181 is a prognostic biomarker for amyotrophic lateral sclerosis

Iddo Magen $^{1,2, \$}$, Nancy Sarah Yacovzada ${ }^{1,2,}$, Eran Yanowski ${ }^{1,2}$, Anna Coenen-Stass ${ }^{3 \#}$, Julian Grosskreutz ${ }^{4,5}$, Ching-Hua $\mathrm{Lu}^{6,7}$ Linda Greensmith ${ }^{3}$, Andrea Malaspina ${ }^{3,6 *}$, Pietro Fratta ${ }^{3 *}$ and Eran Hornstein ${ }^{1,2 *}$

1 Department of Molecular Genetics, Weizmann Institute of Science, Rehovot, Israel 2 Department of Molecular Neuroscience, Weizmann Institute of Science, Rehovot, Israel 3 Department of Neuromuscular Diseases, UCL, Queen Square Institute of Neurology, London, UK

4 Department of Neurology, Jena University Hospital, Jena, Germany

5 Center for Healthy Aging, Jena University Hospital, Jena, Germany

6 Centre for Neuroscience and Trauma, Blizard Institute, Barts and the London School of Medicine and Dentistry, Queen Mary University of London, London, UK.

7 Neurology, School of Medicine, China Medical University and Hospital, Taichung, Taiwan

" Present address: Translational Medicine, Oncology R\&D, AstraZeneca, Cambridge, UK

* To whom correspondence should be addressed: a.malaspina@ucl.ac.uk, p.fratta@ucl.ac.uk or eran.hornstein@weizmann.ac.il

\$ Equal contribution

Abstract word count: 199

Main text word count (excluding references and figure legends): 5600

No. of items (figures, tables): 5, 2

References: 45

One Sentence Summary: plasma miR-181 levels indicate high mortality risk in ALS patients. 


\begin{abstract}
(192 words)
Amyotrophic lateral sclerosis (ALS) is a relentless neurodegenerative syndrome of the human motor neuron system, for which no effective treatment exists. Variability in the rate of disease progression limits the efficacy of ALS clinical trials, suggesting that developing of better biomarkers for prognosis will facilitate therapeutic progress. Here, we applied unbiased nextgeneration sequencing to investigate the potential of plasma cell-free microRNAs as biomarkers of ALS prognosis, in 252 patients with detailed clinical-phenotyping. First, we identified miRNAs, whose plasma levels remain stable over the course of disease in a longitudinal cohort of 22 patients. Next, we demonstrated that high levels of miR-181, a miRNA enriched in neurons of the brain and spinal cord, predicts a $>2$ fold risk of death in discovery cohort (126 patients) and an independent replication cohort (additional 122 patients). miR-181 performance is comparable with the established neurofilament light chain (NfL) biomarker and when combined together, miR$181+\mathrm{NfL}$ establish a novel RNA-protein biomarker pair with superior prediction capacity of ALS prognosis. Therefore, plasma miR-181 predicts ALS disease course, and a novel miRNA-protein biomarker approach, based on miR-181+NfL, boosts precision of patient stratification and may greatly enhance the power of clinical trials.
\end{abstract}




\section{Introduction}

2 Amyotrophic lateral sclerosis (ALS) is a devastating neurodegenerative disorder of the motor

3 neuron system, for which no effective disease-modifying treatment exists. ALS is characterized

4 by a significant variability in progression rates ${ }^{1,2}$, posing a significant challenge for patient

5 stratification in clinical trials. Thus, reliable predictors of disease progression would be invaluable

6 for ALS patient stratification prior to enrolment in clinical trials. Ideal biomarkers should remain

7 stable during the course of disease, be detectable in accessible tissue, and also be easily

8 measurable. To date, intensive research has identified only a few potential blood-based ALS

9 biomarkers ${ }^{3-5}$, including cell-free neurofilaments ${ }^{6-8}$, and pro-inflammatory cytokines ${ }^{9-11}$.

10 Neurofilament light chain (NfL) was the first blood biomarker to aid in predicting ALS progression

11 rate, but further markers are needed to improve stratification and allow for more effective trials.

12 microRNAs (miRNAs) are endogenous non-coding RNAs that are essential for motor neuron

13 survival and have been shown to be globally downregulated in post mortem ALS motor neurons

14 12-14. While circulating miRNA profiles have been previously characterized in ALS ${ }^{15-19}$, the

15 potential of miRNA biomarkers for ALS prognosis, and as readout of disease progression has not

16 been fully explored.

17 Here, we take a hypothesis-free approach by applying next generation sequencing (RNA-seq) to 18 comprehensively study plasma miRNAs in a large cohort of 252 ALS cases. These studies focused

19 our attention on the miR-181 family, which are expressed from two homologs, polycistronic genes,

20 mir-181a-1/b-1 (human chromosome 9) and mir-181a-2/b-2 (human chromosome 1). The mature

21 miR-181 species are functionally identical in silencing a single set of mRNA targets. We reveal

22 that miR-181 levels predict disease progression in large discovery and a replication cohort, and 
23 demonstrate the effectiveness of combining miR-181 with established neurofilament light chain

24 as a prognostic biomarker pair for ALS. 


\section{Results}

\section{Longitudinal study of circulating miRNAs in ALS}

28 In this work, we sought to explore blood-borne miRNAs as potential prognostic biomarkers for

29 ALS. We used unbiased next generation sequencing to investigate, without an a priori bias, the 30 comprehensive landscape of plasma miRNAs in 252 ALS patients, for which documented clinical 31 and demographic information is available (Table 1).

32 A crucial feature for a prognostic biomarker is its stability across the disease course. We therefore

33 initially investigated a longitudinal sample cohort of 22 patients (clinical data in Table 2), with

34 four longitudinal blood samples taken ( $\left.\mathrm{t}_{1}-\mathrm{t}_{4}\right)$ during the course of 30 months (2.5 years). 88 samples

35 (corresponding to the first cohort of 22 patients), were prepared from total plasma RNA, as 36 previously described ${ }^{20}$, and profiled by RNA-seq for miRNA levels. Linear miRNA quantification

37 was achieved via unique 12-nucleotide molecular identifiers (UMIs). miRNAs with $\geq 50$ UMIs in 38 at least $60 \%$ of the samples (>53 out of 88 samples) were considered above noise level. Thus, of 392008 miRNAs aligned to the human genome (GRCh37/hg19), 187 passed the threshold we set (see

40 Table S1). To reduce noisy miRNAs, we next excluded from further analysis 58 miRNAs with

41 high variability (t4/t1 standard error ratio $\geq 0.2$, Figure $1 \mathrm{~A}, \mathrm{y}$-axis). For example, miR-181a-5p 42 variability across individual patients is limited, relative to that of miR $-1-3 p(F$ test for variance $=$ 43 20.9, p<0.0001, Fig. 1B). We identified 125 miRNA candidate biomarkers, whose plasma levels 44 were relatively stable during disease progression in the same sub-cohort of 22 longitudinal samples 45 (Figure 1A, Table S1) that could be tested as candidate prognostic biomarkers. In addition, four 46 miRNAs, whose levels increased during the course of disease, were subjected validation in a 
47 separated replication longitudinal cohort ( $\mathrm{N}=26$ patients) and may serve measures of functional

48 decline over the course of the disease (Figures S1, S2, S3; Table S3).

50 Discovery of circulating miRNAs as potential biomarkers for ALS prognosis

51 For the main interest of the current study in prognosis analysis, we focused on the 125 miRNAs

52 that displayed stable plasma levels over time. These 125 miRNAs were further investigated in a 53 cohort of 252 patients, for which a single blood sample was collected at enrolment. We randomly

54 split the cohort into two sub-cohorts of 126 patients each, with comparable demographic and 55 clinical features (Figure S4).

57 We performed next generation sequencing on the first cohort of 126 patients termed "discovery 58 cohort", holding out an equally-sized "replication cohort" for validation. Out of the 125 candidate 59 miRNAs, we excluded 19 miRNAs, which did not pass the minimal UMI threshold or QC (Figure 60 S5). Optimal cut-off values were determined for 106 miRNAs predictors, for dichotomizing 61 continuous expression levels to binary (high/low), by iterative testing of the capacity to predict 62 patient survival (time elapsed to death, using Evaluate Cutpoints algorithm ${ }^{21}$ ). 19 additional 63 miRNA were excluded at the QC step (methods). Nine of the remaining 87 miRNAs predicted 64 prognosis in a significant manner, when survival was calculated from either onset (defined as first 65 documented symptoms) or enrollment (Figure 2A, B; Table S1). We further tested the prediction 66 capacity of combinations of miRNAs considering this way potential cooperative information in 67 evaluation of all 36 miRNA pairs $\left[\left(\begin{array}{l}9 \\ 2\end{array}\right)=\frac{9 !}{2 !(9-2) !}=36\right] .20$ out of 36 miRNA pairs predicted prognosis 68 comparably or superior to individual miRNAs (logrank $\mathrm{p}$ value $\leq 0.01$, Figure $2 \mathrm{~A}, \mathrm{~B}, \mathrm{~S} 6$, Table 69 S1). 
70 The monthly mortality hazard ratio (HR) was calculated for 9 miRNAs and 20 miRNA-pairs in a

71 multivariate Cox regression analysis, stratified by the disease stage (at enrollment) and age at onset

72 (methods). This analysis allows calculation of an independent hazard ratio for each covariate (i.e.,

73 single miRNA or miRNA pair), while holding the other covariates constant. We report a risk of

74 dying that is almost five times higher with high plasma levels of miR-181 (featuring two sister 75 miRNAs, miR-181a-5p and miR-181b-5p, Figure 2B; hazard ratio $(\mathrm{HR})=4.55,95 \%$ CI: 1.33 -

$7615.6, p=0.016$, Figure $2 C$ ). None of the other features reached a statistically significant signal.

77 Noteworthy, assessment of miR-181 levels as a continuous variable, opposed to categorical one,

78 did not contribute to prediction of mortality hazard.

79 Stepwise feature selection using bootstrap resampling procedure ${ }^{22}$ is a rigorous scheme for the 80 selection of robust survival outcome predictors, that has been used in ALS biomarker research ${ }^{23}$.

81 We therefore orthogonally selected candidate predictors using backward feature elimination, 82 according to Akaike's information criteria (AIC) across 100 bootstrap samples (Figure 2D; Table

83 S1). miR-181 was the only feature satisfying bootstrap criteria (selected $>70 \%$, significant $>85 \%$ ).

84 Taken together, these data identify miR-181 as the best miRNA predictor of survival in ALS 85 patients by both traditional statistics (logrank analysis (Fig. 2A,B), multivariate Cox proportional 86 hazard (Fig 2C) and by bootstrap model selection (Fig 2D).

\section{Validation of circulating miR-181 as biomarker for ALS prognosis}

89 We next tested the capacity of miR-181 to separate survival curves of patients. Kaplan Meier

90 curves revealed clear separation of survival between with high vs low miR-181 subgroups, based

91 on plasma miR-181 levels at enrolment (discovery cohort: logrank chi^ $2=13.6, p=0.0002$, Figure

92 3A; Table S2). The median patient survival associated with low miR-181 was 18.6 months, 
93 compared to 9 months associated with higher miR-181 levels. Thus, plasma miR-181 levels predict

94 a substantial median survival difference of 9.6 months that is equivalent to a $207 \%$ increase in

95 survival length for patients with lower plasma miR-181 levels. Comparable results were obtained

96 when survival length was calculated from disease onset (Figure 3B).

97

98 We next validated our results in an independent cohort of 122 patients, which was held-out until

99 that point. Thus, we assessed discrimination between prognostic groups by miR-181, using the

100 dichotomization miRNA threshold defined in the discovery cohort. Kaplan Meier curve analysis

101 of plasma miR-181 levels in the replication cohort, also revealed clear survival curve separation

102 between subgroups when survival was calculated from enrolment (logrank $\operatorname{chi}^{\wedge} 2=5.2, \mathrm{p}=0.02$,

103 Figure 3C) or onset (logrank chi^2 $=4.4, \mathrm{p}=0.035$, Figure 3D). Finally, we performed analysis on

104248 patients in the combined cohort, from enrolment and from disease onset. Kaplan Meier

105 analysis of plasma miR-181 levels in the combined cohort revealed clear survival curve separation

106 between subgroups (enrolment, logrank chi^ $2=18.5, \mathrm{p}<0.0001$, Figure $3 \mathrm{E}$; onset, logrank chi^ $2=$

107 16.7, p<0.0001, Figure 3F). miR-181 levels were predictive of survival length, regardless of

108 whether patients were treated with Riluzole or not (Figure S7).

109 Accordingly, Cox regression analysis revealed significant hazard ratios from enrolment for high

110 vs. low levels of miR-181 in the discovery cohort (HR 2.17, 95\% CI: $1.25-3.75$, p=0.006, Figure

$1113 \mathrm{G}$ ), the replication cohort (HR 1.76, 95\% CI: $0.97-3.18$, one-tailed $\mathrm{p}=0.03$ ), and the merged

112 cohort (HR 2.09, 95\% CI: 1.48 - 2.94, p<0.001). Likewise, hazard ratios, calculated from onset,

113 were consistent for discovery (HR 2.83, 95\% CI: $1.7-4.7$, p<0.001), replication (HR 1.83, 95\%

114 CI: 1.1-3.0, one-tailed $\mathrm{p}=0.0087)$ and merged (HR 2.21, 95\% CI: $1.56-3.12, \mathrm{p}<0.001)$ cohorts. 
115 In the discovery cohort, miR-181 displayed a 4-fold increase in patients with higher miR-181

116 levels compared to patients with low miR-181 levels $(\mathrm{p}<0.001$, Figure S8A) while in the

117 replication cohort, miR-181 levels increased by 8.5-fold in the high expression bin $(\mathrm{p}=0.009$,

118 Figure S8B). In addition, a modest but statistically significant correlation was found between

119 plasma miR-181 levels and survival length from enrolment or onset (Figure S8C,D; Table S3).

120 We further tested the D50 model- based descriptors, which is derivative of ALSFRS-R and 121 addresses difficulties in characterizing aggression and the individual disease covered by traditional 122 ALS clinical indices ${ }^{24}$. Applying D50 to miR-181 stratification revealed association of high miR-

123181 levels with aggressive disease (time taken to reach half functionality < 32 months), whereas

124 low miR-181 levels are associated with moderate disease (time to half functionality $>57$ months, $125 \mathrm{p}<0.001$, Fig. S9A; Table S3). Such a 25-month gap to losing half functionality might be 126 clinically important. miR-181 levels also increased by $70 \%$ between mean value of patients 127 suffering from aggressive (D50 < 45 months), relative to moderate (D50 > 45 months) disease (t128 test: $\mathrm{p}=0.03$, not shown).

129 miR-181 levels remain stable over time (Figure 1A, B), which is orthogonally supported by the 130 lack of a difference in rD50, a measure of functional decline over the course of disease, between 131 low and high miR-181 levels ( $\mathrm{p}=0.07$, Figure $\mathrm{S} 9 \mathrm{~B}$ ), as well as the lack of correlation between miR132181 levels and rD50 (Figure S9C). Furthermore, miR-181 levels remained stable at early, 133 progressive and late disease stages $(0 \leq \mathrm{rD} 50<0.25 ; 0.25 \leq \mathrm{rD} 50<0.5 ; \mathrm{rD} 50 \geq 0.5$, respectively; 134 ANOVA: $\mathrm{p}=0.15$, Figure S9D). Therefore, miRNA measurements are unlikely to be biased by 135 sampling at different disease stages. Finally, miR-181 levels were not correlated with progression 136 rate, ALSFRS at enrolment or age at onset, and these clinical parameters were comparable between 137 low and high miR-181 levels (Figure S10). 


\section{$138 \quad$ miR-181 is broadly expressed in neurons}

139 To elucidate the tissue source of miR-181 we revisited previously reported Nanostring data ${ }^{25}$.

140 miR-181a-5p is the ninth most abundant miRNA in laser capture micro-dissected human motor

141 neurons of ALS patients and is also fairly abundant in the CNS in general ${ }^{26}$. We further performed

142 fluorescent in situ hybridization with a probe that hybridizes to miR-181a-5p in the mouse motor

143 cortex and the lumbar spinal cord, two regions affected in ALS (Figure 4A, B). Punctate miR-

144 181a-5p signal was found in motor cortex soma and neurites (Figure 4C) and in ventral horn

145 neurons (Figure 4D). Thus, a conceivable source for miR-181 in ALS patients may be motor

146 neurons in the cortex and spinal cord. The presence of miR-181 in neurites suggests that it could

147 be an RNA marker of axonal damage, resembling the suggested axonal origin of protein

148 biomarkers, such as $\mathrm{NfL}^{27}$.

150 miR-181 \& NfL establish a cooperative miRNA-protein biomarker in prediction of ALS

151 prognosis

152 We have previously shown that neurofilament light chain (NfL) can stratify ALS patients by their

153 survival length ${ }^{28}$. In the current cohort, we assayed NfL in all plasma samples with by single

154 molecule array (Simoa) immunoassay. 243 of the 248 SIMOA samples were technically

155 successful. A Cox proportional hazard analysis revealed that high plasma NfL predicts higher risk

156 of death (from enrollment HR 2.09, 95\% 1.49 - 2.94, p<0.001, concordance index (C-index) 0.59,

157 Akaike's information criteria (AIC) 2083, or from onset HR 2.26, 95\% $1.73-2.96$, p<0.001, C-

158 index 0.62 , AIC 2060 Figure 5A, B), as previously reported ${ }^{28-30}$. The performance of miR-181 in

159 predicting risk of death is comparable with that of NfL (from enrollment HR 2.03, 95\% CI: 1.45 
$160-2.85, \mathrm{p}<0.001, \mathrm{C}$-index 0.56, AIC 2096, or from onset HR 2.07, 95\% CI: $1.6-2.7, \mathrm{p}<0.001, \mathrm{C}-$

161 index 0.56, AIC 2081, Figure 5A, B).

162 We then tested a combined predictor based on both NfL and miR-181, creating a binary protein-

163 miRNA feature "NfL+miR181". An interaction variable based on both NfL and miR-181, yielded

164 higher risk of death than each one of single markers on its own (from enrolment, HR 2.46, 95\%

165 CI: $1.87-3.24$, p <0.001, C-index 0.61, AIC 2071, or from onset: HR 2.7, 95\% CI: $2.05-3.56$, $166 \mathrm{p}<0.001$, C-index 0.63, AIC 2046, Figure 5A, B). Therefore, miR-181 and NfL display comparable 167 capacities, as single estimators of death risk, in patients with ALS. However, together the miRNA168 protein pair displays a cooperative predictive value. Furthermore, we employed the continuous 169 values of miR-181 and NfL, which were standardized with respect to reference values of healthy 170 controls. In this analysis, miR-181 exhibited a higher risk of death than NfL, by Cox proportional 171 hazard analysis. Moreover, a predictor that is based on the sum of NfL and miR-181 z-scores, 172 predicted a higher risk of death and had better goodness of fit than NfL alone in the merged cohort 173 (Figure S11).

174 We then stratified samples into tertiles, according to NfL levels, which exhibits different survival 175 length ${ }^{28}$, (Figure 5C, D). Interestingly, in the range of intermediate NfL levels, the additional 176 stratification by miR-181 separated this sub-cohort in two, as revealed by KM analysis (from 177 enrollment logrank chi^2 $=41.5, \mathrm{p}<0.0001$, from onset, logrank chi^2 $=51.1, \mathrm{p}<0.0001$, Figure 178 5E, F). Cox regression analysis on miR-181 levels in the low, intermediate, and high NfL tertiles 179 revealed a higher risk of dying with higher miR-181 plasma levels in the middle and high tertiles 180 (from enrolment: HR 2.0, 95\% CI, $1.1-3.6, \mathrm{p}=0.03$, Figure 5G), but not in the low tertile (HR $1810.96,95 \% \mathrm{CI}, 0.55-1.66, \mathrm{p}=0.9$ ). Similarly, when calculated from disease onset, higher miR-181 182 levels predicted a higher risk of dying for patients within the range of intermediate NfL tertile (HR 
$1832.37,95 \%$ CI, 1.4-4.02, $\mathrm{p}=0.001$, Figure 5H) and a modest added risk in the high NfL tertile (HR $1841.66,95 \% \mathrm{CI}, 1.0-2.7, \mathrm{p}=0.04)$. Therefore, miR-181 may be valuable in particular at the range of 185 intermediate NfL values, where it can accurately call a 18 months difference in median prognosis 186 that cannot be identified by measurements of NfL alone.

187 We tested the potential correlation of miR-181 with other molecular biomarkers that are under 188 investigation, neurofilaments, TNF, creatinine and creatine kinase in the same cohort. Notably, 189 miR-181 levels did not correlate with the levels of other plasma biomarkers, (Figure S12; Table 190 S3), suggesting it works via an alternative mechanism.

191 Finally, we were interested in the relationship of bimolecular blood predictors and established 192 clinical features of the disease. Thus, we performed multivariate Cox analysis using the combined 193 predictor NfL+miR181 along with eight other clinical features that were previously shown to be 194 informative (age of onset, forced vital capacity, diagnostic delay, enrolment progression rate, site 195 of onset, El Escorial's definite ALS, cognitive dysfunction and C9orf72 genetics) ${ }^{23}$. High $196 \mathrm{NfL}+\mathrm{miR} 181$ predicted a risk of dying that was 3-4.6 times higher (from enrolment HR 3.06, 95\%

197 CI: $1.5-6.24, p=0.002$; from onset HR 4.63, 95\% CI: $1.98-10.82, p<0.001$, Figure 5I, J).

198 We have also performed a digital PCR study to quantify miR-181 RNA molecule concentration in 199 human plasma. Unique molecular identifiers (UMIs) in sequencing correlated to absolute miRNA 200 copies by digital PCR with Pearson $\mathrm{R}^{2} 0.97$ (327, 389, 523, 688 UMIs corresponding to 4020, $2015760,6960,9540 \mathrm{miR}-181$ RNA molecules /microliter of human plasma). This analysis further 202 suggests that the threshold of miR-181, when utilized with NfL as biomarker pair, is at 203 approximately 5340 RNA molecules /microliter of human plasma).

204 Finally, the predicted survival curve, with the miRNA-protein predictor NfL+miR181, is closer to 205 the observed (real) survival curve, than survival curve approximated by the multivariate Cox 
206 model with eight established clinical features. Together, miR-181 stands on its own as a powerful

207 prognostic marker for ALS. Furthermore, utilization of miR-181 in concert with an established 208 protein biomarker, NfL, is more accurate than either alone. 


\section{Discussion}

210 In this study, we report the results of one of the most elaborated small RNA-seq studies, undertaken

211 to date in neurodegeneration research. We show that in ALS, miRNAs appear to be mostly

212 unchanged longitudinally during disease (Figure 1), whereas increase in miR-423/484/92a/b levels

213 during disease course could contribute to monitoring of disease progression (Figure S2,3).

214 Importantly, high miR-181 levels predicted shortened survival in two ALS cohorts. miR-181 is

215 encoded from a human gene that has seen local duplication to a bi-cistronic miR-181a and miR-

$216181 \mathrm{~b}$ within the same transcriptional unit and additional genomic duplications that results in three

217 homologs across the human genome. miR-181a and miR-181b are functionally identical, silence

218 the same target set, and are co-expressed from the same gene. Although equivalent, the

219 simultaneous consideration of both RNAs provides superior sensitivity as a predictor of ALS

220 prognosis and progression (Figure 2). miR-181 ability to predict prognosis of patients with ALS

221 was validated in a replication cohort (Figure 3). The fact that miR-181 levels stay stable during

222 the course of disease, suggests a constant process underlying their generation and clearance rates.

223 miR-181 species are expressed in the brain and spinal cord, including in cortical and spinal motor

224 axons and soma (Figure 4) and their transport and biogenesis is regulated in neuronal axons ${ }^{31}$.

225 Therefore, it may be that the utility of miR-181 as a prognostic biomarker in ALS is linked to being

226 spilled off dying axons, somewhat reminiscent of NfL, which is a neuronal cytoskeletal protein.

227 Accordingly, we demonstrate that miR-181 and NfL serve separately as predictors of ALS

228 prognosis, with comparable predictive capacity (Figure 5). Furthermore, miR-181 measurement

229 can enhance the prognostic value of NfL and a joint miRNA-protein measure may compute

230 prognosis more precisely than any of the circulating biomolecules on their own. Specifically, we

231 show that miR-181 levels were of predictive value particularly when NfL values are intermediate, 
232 and the combination of miR-181 and NfL is able to discriminate fast and slow progressors in this

233 group. Stratification based on progression rate is important in clinical trials to balance treatment

234 and placebo groups. Indeed, certain trials have focused on ALS fast progressors in order to obtain

235 outcomes that are more reliable. Our proposed combination of miR-181 and NfL as a prognostic

236 biomarker would enhance our ability to predict ALS progression and thereby facilitate recruitment

237 to clinical trials. Therefore, the development of a miR-181-based biomarker opens a new horizon

238 for a combinatorial protein-RNA biomarker system for ALS prognosis and encourages testing the

239 value of orthogonal multi-omic platforms for additional biomarker endpoints.

240 We also found miR-181 expressed in the brain and the spinal cord, and suggest that plasma miR-

241181 originates in part from the central nervous system, reminiscent of NfL. While miR-181 was

242 reported in the cerebrospinal fluid of ALS patients, and might contribute to ALS diagnosis ${ }^{19}$, it is

243 also abundant in hematopoietic tissues ${ }^{32}$, which also contribute to its presence in the plasma. That

244 miR-181 levels do not correlate with clinical features or several other circulating biomolecules

245 may perhaps reflect different facets of the medical condition or disparate underlying mechanisms.

247 Several additional factors contribute to the potential clinical impact of miR-181 quantification.

248 When neurofilaments are released into the circulation, endogenous antibodies with variable

249 degrees of affinity and avidity are formed against them. These endogenous antibodies limit

250 neurofilament detection twofold: by initiating antigen-clearing effect and by interfering with

251 immunoassays ${ }^{28,30,33-35}$. In such cases, the value of miR-181 may be even more pronounced, since

252 miRNA feature negligible immunogenicity, compared to proteins and miRNA detection is not

253 dependent on antibody-based immune-detection assays. In addition, real-world patient profiles 
254 indicate that most ALS patients are in the middle tertile neurofilament level, ${ }^{30}$ the region of the 255 NfL spectrum, where enhanced sensitivity provided by miR-181 will be particularly useful.

256 Taken together, miR-181 emerges as an ALS prognostication biomarker that can be developed in 257 combination with NfL to improve the accuracy of patient stratification in clinical trials. 


\section{Online Methods}

\section{Standard protocol approvals, registrations, and patient consents}

260 The study included a cohort with 252 patients with ALS from the ALS biomarker study. Patients

261 were diagnosed with ALS according to standard criteria by experienced ALS neurologists ${ }^{36}$. Two

262 additional control cohorts were of 103 adult individuals and of 73 adult individuals. All

263 participants provided written consent (or gave verbal permission for a carer to sign on their behalf)

264 to be enrolled in the ALS biomarkers study if they met inclusion criteria until the desired sample

265 size was reached (consecutive series). Ethical approval was obtained from East London and the

266 City Research Ethics Committee 1 (09/H0703/27).

\section{Study design}

268 We determined the sample size by doubling this number calculated by the following power 269 analysis: 120 ALS patients are needed to obtain a hazard ratio of 3 with a power of $99 \%$ and a p270 value of 0.01 . A full cohort of 252 patients was randomly split into a discovery and replication 271 cohorts with comparable clinical characteristics, each with 126 patients. Phenotypic data on de272 identified patients was separated and blinded during steps of the molecular analysis. Disease 273 severity was assessed with the revised ALS Functional Rating Scale (ALSFRS-R) ${ }^{38}$, and 274 progression rate at enrolment (i.e. first blood draw) was calculated as follows: (48 - enrolment 275 ALSFRS-R)/time (in months) from symptom onset to enrolment. Progression was also modeled 276 using the D50 model which fits a sigmoid decay across all available ALSFRS-R scores ${ }^{39,40}$. Use 277 of Riluzole (or not) at the time of sampling was recorded. Blood was collected by venipuncture in 278 EDTA tubes, and plasma was recovered from the whole blood sample by centrifugation and stored 279 at $-80^{\circ} \mathrm{C}$ until performing downstream assays (RNA-seq and SIMOA for NfL). 


\section{Longitudinal cohort analysis}

283 Serial plasma samples and clinical information were obtained, on average, every 2 to 4 months

284 from 48 patients with ALS. No selection criteria were applied to individuals with ALS sampled

285 longitudinally, other than their willingness to donate further samples. Longitudinal analysis of 286 miRNAs was first performed on samples from 22 patients, in an unbiased manner by next 287 generation RNA sequencing. Results were tested on a replication longitudinal cohort of 26 patients 288 by an orthogonal method of quantitative real time PCR. Symptom onset was defined as first 289 patient-reported weakness.

\section{Small RNA next generation sequencing}

292 Total RNA was extracted from plasma using the miRNeasy micro kit (Qiagen, Hilden, Germany) 293 and quantified with Qubit fluorometer using RNA broad range (BR) assay kit (Thermo Fisher

294 Scientific, Waltham, MA). For small RNA next generation sequencing (RNA-seq), libraries were 295 prepared from $7.5 \mathrm{ng}$ of total RNA using the QIAseq miRNA Library Kit and QIAseq miRNA

296 NGS 48 Index IL (Qiagen), by an experimenter who was blinded to the identity of samples. 297 Samples were randomly allocated to library preparation and sequencing in batches. The 298 longitudinal ALS study samples were sequenced in one batch to avoid batch-induced biases in 299 interpretation of longitudinal changes (analyzed in Figure 1). Precise linear quantification of 300 miRNA is achieved by using unique molecular identifiers (UMIs), of random 12-nucleotide after $3013^{\prime}$ and 5 ' adapter ligation, within the reverse transcription primers ${ }^{20}$. cDNA libraries were 302 amplified by PCR for 22 cycles, with a 3' primer that includes a 6-nucleotide unique index, 303 followed by on-bead size selection and cleaning. Library concentration was determined with Qubit 304 fluorometer (dsDNA high sensitivity assay kit ; Thermo Fisher Scientific, Waltham, MA) and 
305 library size with Tapestation D1000 (Agilent). Libraries with different indices were multiplexed 306 and sequenced on NextSeq 500/550 v2 flow cell or Novaseq SP100 (Illumina), with 75bp single

307 read and 6bp index read. Fastq files were de-multiplexed using the user-friendly transcriptome 308 analysis pipeline (UTAP) ${ }^{41}$. Human miRNAs, as defined by miRBase ${ }^{42}$, were mapped using

309 Geneglobe (Qiagen). Sequencing data normalized with DESeq2 package ${ }^{43}$ under the assumption

310 that miRNA counts followed negative binomial distribution and data were corrected for the library

311 preparation batch in order to reduce its potential bias. 103 individuals without sign of neurological

312 disease, were enrolled in a control cohort for miR-181 levels.

\section{Selecting candidate miRNA and miRNA pairs for prognostic analysis}

315 The pipeline is succinctly described in Figure S5. 2008 miRNAs were aligned to the genome in 316 the longitudinal study and out of them, 187 miRNAs that exhibited $>50$ UMI counts in $60 \%$ of the 317 samples and non-zero counts in all samples, were included in further analysis. 125 out of these 187

318 miRNAs were longitudinally stable with low inter-individual variability (blue features in Figure

319 1A). In the discovery cohort, 106 out of these 125 miRNAs passed a filtering criterion of average

320 UMI counts $>100$ across all samples and non-zero counts, and were analyzed for prognosis

321 differences between low and high level in the discovery cohort. Then, the miRNAs predictors were

322 transformed from a continuous expression level to binary predictors (high/low), when the optimal

323 dichotomization cut-off values were determined by iterative logrank analysis on all possible

324 sample distributions for the remaining 106 miRNAs ${ }^{21} .19$ miRNAs were further excluded after

325 additional QC if different miRNAs of the same family provided conflicting prognosis predictions,

326 e.g. miR-27a predicted beneficial prognosis and miR-27b a detrimental prognosis). A logrank test,

327 to compare survival distributions was performed for the remaining 87 miRNAs and null hypothesis 
328 significance testing ( $\mathrm{p}$ values) for prognosis differences as demonstrated in Figure 2. Nine out of

32987 miRNAs displayed logrank $\mathrm{p} \leq 0.01$. All 36 combinatorial pairs of these 9 miRNAs $(9 * 8 / 2=36)$,

330 were also subjected to logrank test to test cooperative prediction, via multiplication of the levels

331 of two single miRNAs and then transforming them to a binary (high/low) predictors, as done for

332 single miRNA predictors. 20 (out of 36) miRNA pairs displayed logrank $\mathrm{p} \leq 0.01$. A total of 29

333 candidate prognostic biomarkers ( 9 miRNAs and 20 miRNA pairs) were then examined under

334 bootstrap feature selection.

\section{Model selection: AIC-based backward feature selection by bootstrap resampling}

336 Feature selection by bootstrap resampling was performed on a full Cox model of 29 features (9

337 single candidate miRNAs and 20 miRNA pairs) using stepwise backward elimination based on

338 Akaike information criterion (AIC) ${ }^{22}$. The Cox regression coefficients and standard errors are

339 estimated in the full model (null model), including all variables under consideration, and at each

340 step a single feature is eliminated until no significant improvement in AIC is obtained. The

341 procedure is repeated for 100 bootstrap samples, that were randomly drawn from the original

342 cohort $(n=126)$. Within each bootstrap sample, a Cox model is developed and a list of selected

343 features that optimize AIC is obtained. Candidate biomarkers are then ranked according to the

344 proportion of bootstrap samples in which they were selected as best predictors, and the proportion

345 of bootstrap samples where their Cox coefficient was significant (at significance level 0.05). We

346 considered the following criteria for selecting the final prognostic biomarkers from the bootstrap

347 resampling procedure: features that were selected $>70 \%$ of bootstrap samples and were statistical

348 significance in $>85 \%$ of the bootstrap samples in which they were selected.

349 Only a single predictor fulfilled these criteria, miR-181. A univariate Cox model of miR-181,

350 stratified by ALSFRS slop and age of onset is then assessed on discovery and replication cohort. 
351 A numerical threshold of 71,000 UMIs, which was found as optimal by Evaluate Cutpoints ${ }^{21}$ in

352 the discovery cohort $(\mathrm{N}=126)$, separated between sub-threshold patients $(\mathrm{N}=104)$ and supra-

353 threshold patients $(\mathrm{N}=22)$. Same threshold was used in an independent replication cohort, whereby

3544 out of 126 samples, with borderline miR-181 levels, were excluded. Joint analysis with the same

355 threshold was further conducted on the combined cohort of 248 patients.

\section{Polymerase chain reaction assays}

357 Quantitative real time PCR (qPCR) of miR-423/484/92a/92b, performed with Taqman advanced

358 miRNA assay probes (Thermo Fisher) with the following probes: hsa-miR-423-5p (Assay ID:

359 478090_mir); hsa-miR-484 (Assay ID: 478308); hsa-miR-92a-3p (Assay ID: 477827); hsa-miR-

360 92b-3p (Assay ID: 477823). hsa-miR-140-3p (Assay ID: 477908) and hsa-miR-185-5p (Assay ID:

361 477939) were selected as normalizers, based on stable plasma levels in the longitudinal cohort,

362 described in Figure 1: (1) basemean expression between 500-3,000; (2) coefficient of variation $\leq$

363 0.35.cDNA Synthesis Kit (Applied Biosystems) was used for cDNA reverse transcription (10 ng 364 input) and run on a StepOnePlus (Applied Biosystems). Data compared between samples at

365 enrolment $\left(\mathrm{t}_{1}\right)$ and corresponding follow-up sample $\left(\mathrm{t}_{2}\right)$ by one-tailed paired t-test. Digital droplet

366 PCR (ddPCR) was performed using the hsa-miR-181a and hsa-miR-181b probes (Taqman assay

367 ID: 000480, 001098, respectively, Thermo Fisher Scientific). Mix (5 $\mu \mathrm{L}$ of cDNA $11 \mu \mathrm{L}$ ddPCR 368 supermix (Bio-Rad), $1 \mu \mathrm{L} \times 20$ TaqMan Assay, $5 \mu \mathrm{L} \mathrm{H}_{2} \mathrm{O}$ ) was gently vortexed, droplets were 369 generated in QX100/QX200 with DG8 cartridges (Bio-Rad) and put into 96-well in C1000 370 thermocycler (Bio-Rad) for a protocol: $95^{\circ} \mathrm{C}, 10$ minutes $(1$ cycle $), 60^{\circ} \mathrm{C}$ annealing/extension step, 3711 minute followed by $94^{\circ} \mathrm{C}$ melting step, 30 seconds ( 39 cycles), and a final stage of $98^{\circ} \mathrm{C}, 10$ 372 minutes followed by holding at $12^{\circ} \mathrm{C}$. Plates read on the QX200 droplet and analyzed by 
373 QuantaSoft software (Bio-rad) after setting a FAM threshold based on the 'no template' negative 374 control fluorescence histogram.

375 
377 The quantitative determination of NfL in human plasma was undertaken by Single Molecule Array 378 technology using a digital immunoassay Simoa HD-1 Analyzer (Quanterix, Lexington, MA).

379 Standards, primary and secondary antibodies, detection range including lower and upper limits of 380 detection were specified by manufacturer (Simoa Nf-L Advantage Kit-102258, Quanterix). An 381 equal volume was loaded for all samples in study. NfL threshold concentrations were defined by 382 cohort tertiles: $N f L<59 \mathrm{pg} / \mathrm{ml}$ for the lower tertile (81 patients), $59-109.8 \mathrm{pg} / \mathrm{ml}$ for the middle 383 tertile (81 patients), or $>109.8 \mathrm{pg} / \mathrm{ml}$ for the higher tertile (81 patients). 73 individuals without 384 sign of neurological disease (50 from the National Hospital for Neurology in London, UK, and 23 385 from the University Hospital in Padova, Italy) ${ }^{37}$ were defined as NfL control cohort.

\section{RNA in situ hybridization}

Mouse studies, performed in accordance with institutional guidelines and IACUC. Adult mice were deeply anesthetized (10\% ketamine, $2 \%$ xylazine in PBS, $0.01 \mathrm{ml} /$ gram body weight) and 390 intracardially perfused with $10 \mathrm{ml}$ of PBS, followed by $40 \mathrm{ml}$ of $4 \%$ Paraformaldehyde. Brains and 391 spinal cords were dissected, fixed in fresh 4\% PFA at room temperature for $24 \mathrm{hrs}$., dehydrated in 392 graded ethanol series, cleared with ethanol / histoclear (1:1 vol. / vol.) and then in histoclear 393 (National Diagnostics) and embedded in paraffin. $4 \mu \mathrm{m}$ microtome sections were mounted onto 394 Superfrost plus slides and deparaffinized. miRNA in-situ hybridization performed with hsa-miR395 181a-5p probe (VM1-10255-VCP, ViewRNA Tissue Assay, Thermo-Fisher Scientific), counterstained with DAPI and mounted with ProLong Gold (Molecular Probes, P36934). Adjacent

397 sections were taken for cresyl violet (Nissl) staining. Micrograph acquisition performed on 398 Dragonfly Spinning disc confocal system (Andor Technology PLC) with Leica Dmi8 Inverted 
399 microscope (Leica GMBH) with 10X (air) and 63X (glycerol) objectives equipped with sCMOS 400 Zyla (Andor) 2048X2048 Camera. DAPI (Excitation 405nm, emission 450/50nm, 100ms);

401 ViewRNA probe (excitation 561nm emission 620/60nm, 200ms). Background and Shading 402 Correction was performed using BaSIC ${ }^{44}$.

\section{Analysis of the combination of $\mathrm{NfL}$ and miR-181 as prognostic factors}

404 Logical operators developed to define a combined miRNA-protein predictor for ALS prognosis:

405 If $(N f L<59 \mathrm{pg} / \mathrm{ml})$ or $(\mathrm{NfL} 59-109.8 \mathrm{pg} / \mathrm{ml}$ AND miR-181 $<39,300 \mathrm{UMIs})=N f L+m i R 181=0$

406 If $(N f L>109.8 \mathrm{pg} / \mathrm{ml})$ or $(N f L 59-109.8 \mathrm{pg} / \mathrm{ml}$ AND miR-181 $>39,300 \mathrm{UMIs})=N f L+m i R 181=$ $407 \quad 1$

408 Analysis of clinical features as prognostic factors

409 A subset of 75 patients out of the all 252 participates of the study, held the complete clinical 410 information sufficient to perform multivariate Cox analysis with $N f L+m i R 181$ eight clinical 411 covariates (diagnostic delay, forced vital capacity, C9ORF72 genetics, progression rate at 412 enrolment, cognitive dysfunction, age at onset, bulbar onset and definite ALS by El-Escorial 413 criteria) that were described in ${ }^{23}$.

\section{Statistical analysis}

415 In longitudinal cohort, $\mathrm{p}$ values were calculated by Wald test ${ }^{43,45}$ and adjusted for multiple testing 416 according to Benjamini and Hochberg ${ }^{46}$. Logrank Mantel-Cox test was used for Kaplan-Meier 417 survival estimators and a fixed date was used to censor data for survival analysis. Optimal 418 dichotomization cut-off values of miRNA levels determined using Evaluate Cutpoints ${ }^{21}$. 419 Multivariate or univariate Cox proportional hazard analyses were used to calculate mortality 420 hazard ratios, with molecular and phenotypic features as covariates. Cox model goodness of fit 421 was determined by $\mathrm{C}$-index ${ }^{47}$. For longitudinal miRNA expression by qPCR, one-tailed paired $\mathrm{t}$ - 
422 test was used. Outliers were detected by Grubbs test ${ }^{48}$ and excluded from analysis. Tests were run

423 in R Project for Statistical Computing environment ${ }^{49}$ and graphs were generated with Prism 5

424 (GraphPad Software, San Diego, California, USA).

425 


\section{Acknowledgements}

427 We thank Vittoria Lombardi (UCL) for technical assistance, and Iddo Ben-Dov from Hadassah 428 Hebrew University Medical Center, Jerusalem, Israel, for advice on statistics. We acknowledge 429 patients for their contribution and all the ALS biomarkers study co-workers and their contribution 430 to the biobanking project, which has made this study possible (REC 09/H0703/27). We also thank 431 the North Thames Local Research Network (LCRN) for its support and life science editors for 432 editorial assistance. EH is the Mondry Family Professorial Chair and Head of the Nella and Leon 433 Benoziyo Center for Neurological Diseases. Imaging performed at de Picciotto Cancer Cell 434 Observatory, in memory of Wolfgang and Ruth Lesser.

\section{Funding}

436 This research was supported by the following grants: Motor Neuron Disease Association (MNDA 437 \#839-791). Research at Hornstein lab is supported by CReATe consortium and ALSA (program: 438 'Prognostic value of miRNAs in biofluids from ALS patients'), RADALA Foundation; AFM 439 Telethon (20576); Weizmann - Brazil Center for Research on Neurodegeneration at Weizmann 440 Institute of Science; the Minerva Foundation with funding from the Federal German Ministry for 441 Education and Research, ISF Legacy Heritage Fund 828/17; Israel Science Foundation 135/16; $442392 / 21 ; 393 / 21 ; 3497 / 21$. Target ALS 118945; Thierry Latran Foundation for ALS Research. the 443 European Research Council under the European Union's Seventh Framework Programme 444 (FP7/2007-2013)/ERC grant agreement number 617351; ERA-Net for Research Programmes on 445 Rare Diseases (eRARE FP7) via Israel Ministry of Health; Dr. Sydney Brenner and friends, 446 Edward and Janie Moravitz, A. Alfred Taubman through IsrALS, Yeda-Sela, Yeda-CEO, Israel

447 Ministry of Trade and Industry; Y. Leon Benoziyo Institute for Molecular Medicine, Kekst Family 448 Institute for Medical Genetics; David and Fela Shapell Family Center for Genetic Disorders 
449 Research; Crown Human Genome Center; Nathan, Shirley, Philip and Charlene Vener New 450 Scientist Fund; Julius and Ray Charlestein Foundation; Fraida Foundation; Wolfson Family 451 Charitable Trust; Adelis Foundation; Merck (United Kingdom); M. Halphen; and Estates of F. 452 Sherr, L. Asseof, L. Fulop. From:benatPF is supported by an MRC/MND LEW Fellowship and by 453 the NIHR UCLH BRC. JG was supported in the JPND framework ONWebDUALS and LG is the

454 Graeme Watts Senior Research Fellow supported by the Brain Research Trust. NSY was supported 455 by the Israeli Council for Higher Education (CHE) via the Weizmann Data Science Research 456 Center. IM was supported by Teva Pharmaceutical Industries Ltd. as part of the Israeli National 457 Network of Excellence in Neuroscience (NNE, fellowship 117941).

459 Competing interests

460 The authors state that they have no competing interests.

462 Data availability

463 Source data for figures are provided in supplementary tables. Fastq.gz files with raw sequencing 464 data, text files with raw read counts, excel files with processed read counts and $\mathrm{R}$ codes are 465 available as GSE 168714 in gene expression omnibus (GEO). 


\section{References}

1. Munsat, T.L., Andres, P.L., Finison, L., Conlon, T. \& Thibodeau, L. The natural history of motoneuron loss in amyotrophic lateral sclerosis. Neurology 38, 409-413 (1988).

2. Ravits, J.M. \& La Spada, A.R. ALS motor phenotype heterogeneity, focality, and spread: deconstructing motor neuron degeneration. Neurology 73, 805-811 (2009).

3. Benatar, M., et al. ALS biomarkers for therapy development: State of the field and future directions. Muscle Nerve 53, 169-182 (2016).

4. Chipika, R.H., Finegan, E., Li Hi Shing, S., Hardiman, O. \& Bede, P. Tracking a Fast-Moving Disease: Longitudinal Markers, Monitoring, and Clinical Trial Endpoints in ALS. Front Neurol 10, 229 (2019).

5. Verber, N.S., et al. Biomarkers in Motor Neuron Disease: A State of the Art Review. Front Neurol 10, 291 (2019).

6. Rossi, D., et al. CSF neurofilament proteins as diagnostic and prognostic biomarkers for amyotrophic lateral sclerosis. J Neurol 265, 510-521 (2018).

7. Goncalves, M., et al. Phosphoneurofilament heavy chain and vascular endothelial growth factor as cerebrospinal fluid biomarkers for ALS. Amyotrophic lateral sclerosis \& frontotemporal degeneration 18, 134-136 (2017).

8. Lu, C.H., et al. Plasma neurofilament heavy chain levels and disease progression in amyotrophic lateral sclerosis: insights from a longitudinal study. J Neurol Neurosurg Psychiatry 86, 565-573 (2015).

9. Gonzalez-Garza, M.T., Martinez, H.R., Cruz-Vega, D.E., Hernandez-Torre, M. \& Moreno-Cuevas, J.E. Adipsin, MIP-1b, and IL-8 as CSF Biomarker Panels for ALS Diagnosis. Dis Markers 2018, 3023826 (2018).

10. Prado, L.G.R., et al. Longitudinal assessment of clinical and inflammatory markers in patients with amyotrophic lateral sclerosis. J Neurol Sci 394, 69-74 (2018).

11. Lu, C.H., et al. Systemic inflammatory response and neuromuscular involvement in amyotrophic lateral sclerosis. Neurol Neuroimmunol Neuroinflamm 3, e244 (2016).

12. Haramati, S., et al. miRNA malfunction causes spinal motor neuron disease. Proc Natl Acad Sci U S A 107, 13111-13116 (2010).

13. Emde, A., et al. Dysregulated miRNA biogenesis downstream of cellular stress and ALS-causing mutations: a new mechanism for ALS. EMBO J 34, 2633-2651 (2015).

14. Eitan, C. \& Hornstein, E. Vulnerability of microRNA biogenesis in FTD-ALS. Brain Res (2016).

15. Takahashi, I., et al. Identification of plasma microRNAs as a biomarker of sporadic Amyotrophic Lateral Sclerosis. Mol Brain 8, 67 (2015).

16. de Andrade, H.M., et al. MicroRNAs-424 and 206 are potential prognostic markers in spinal onset amyotrophic lateral sclerosis. J Neurol Sci 368, 19-24 (2016).

17. Sheinerman, K.S., et al. Circulating brain-enriched microRNAs as novel biomarkers for detection and differentiation of neurodegenerative diseases. Alzheimers Res Ther 9, 89 (2017).

18. Dobrowolny, G., et al. A longitudinal study defined circulating microRNAs as reliable biomarkers for disease prognosis and progression in ALS human patients. Cell death discovery 7, 4 (2021).

19. Benigni, M., et al. Identification of miRNAs as Potential Biomarkers in Cerebrospinal Fluid from Amyotrophic Lateral Sclerosis Patients. Neuromolecular medicine 18, 551-560 (2016).

20. Coenen-Stass, A.M.L., et al. Evaluation of methodologies for microRNA biomarker detection by next generation sequencing. RNA Biol 15, 1133-1145 (2018).

21. Ogłuszka, M., Orzechowska, M., Jędroszka, D., Witas, P. \& Bednarek, A.K. Evaluate Cutpoints: Adaptable continuous data distribution system for determining survival in Kaplan-Meier estimator. Computer Methods and Programs in Biomedicine 177, 133-139 (2019). 
22. Austin, P.C. \& Tu, J.V. Bootstrap Methods for Developing Predictive Models. The American Statistician 58, 131-137 (2004).

23. Westeneng, H.J., et al. Prognosis for patients with amyotrophic lateral sclerosis: development and validation of a personalised prediction model. The Lancet. Neurology 17, 423-433 (2018).

24. Steinbach, R., et al. Disease aggressiveness signatures of amyotrophic lateral sclerosis in white matter tracts revealed by the D50 disease progression model. Human brain mapping 42, 737-752 (2021).

25. Reichenstein, I., et al. Human genetics and neuropathology suggest a link between miR-218 and amyotrophic lateral sclerosis pathophysiology. Science Translational Medicine 11, eaav5264 (2019).

26. Ludwig, N., et al. Distribution of miRNA expression across human tissues. Nucleic Acids Research 44, 3865-3877 (2016).

27. Khalil, M., et al. Neurofilaments as biomarkers in neurological disorders. Nature Reviews Neurology 14, 577-589 (2018).

28. Lu, C.H., et al. Neurofilament light chain: A prognostic biomarker in amyotrophic lateral sclerosis. Neurology 84, 2247-2257 (2015).

29. Poesen, K. \& Van Damme, P. Diagnostic and Prognostic Performance of Neurofilaments in ALS. Front Neurol 9, 1167 (2018).

30. Benatar, M., et al. Validation of serum neurofilaments as prognostic and potential pharmacodynamic biomarkers for ALS. Neurology 95, e59-e69 (2020).

31. Corradi, E., et al. Axonal precursor miRNAs hitchhike on endosomes and locally regulate the development of neural circuits. EMBO J 39, e102513 (2020).

32. Chen, C.-Z., Li, L., Lodish, H.F. \& Bartel, D.P. MicroRNAs Modulate Hematopoietic Lineage Differentiation. Science 303, 83 (2004).

33. Benatar, M., Wuu, J., Andersen, P.M., Lombardi, V. \& Malaspina, A. Neurofilament light: A candidate biomarker of presymptomatic amyotrophic lateral sclerosis and phenoconversion. Ann Neurol 84, 130-139 (2018).

34. Puentes, F., et al. Immune reactivity to neurofilaments and dipeptide repeats in ALS progression. bioRxiv, 2020.2002.2025.965236 (2020).

35. Puentes, F., et al. Immune reactivity to neurofilament proteins in the clinical staging of amyotrophic lateral sclerosis. J Neurol Neurosurg Psychiatry 85, 274-278 (2014).

36. Brooks, B.R., Miller, R.G., Swash, M., Munsat, T.L. \& World Federation of Neurology Research Group on Motor Neuron, D. El Escorial revisited: revised criteria for the diagnosis of amyotrophic lateral sclerosis. Amyotroph Lateral Scler Other Motor Neuron Disord 1, 293-299 (2000).

37. Lombardi, V., et al. Muscle and not neuronal biomarkers correlate with severity in spinal and bulbar muscular atrophy. Neurology 92, e1205-e1211 (2019).

38. Cedarbaum, J.M., et al. The ALSFRS-R: a revised ALS functional rating scale that incorporates assessments of respiratory function. BDNF ALS Study Group (Phase III). J Neurol Sci 169, 13-21 (1999).

39. Poesen, K., et al. Neurofilament markers for ALS correlate with extent of upper and lower motor neuron disease. Neurology 88, 2302-2309 (2017).

40. Prell, T., et al. Reaction to Endoplasmic Reticulum Stress via ATF6 in Amyotrophic Lateral Sclerosis Deteriorates With Aging. Front Aging Neurosci 11, 5 (2019).

41. Kohen, R., et al. UTAP: User-friendly Transcriptome Analysis Pipeline. BMC bioinformatics 24, 154 (2019).

42. Kozomara, A. \& Griffiths-Jones, S. miRBase: annotating high confidence microRNAs using deep sequencing data. Nucleic Acids Res 42, D68-73 (2014). 
43. Love, M.I., Huber, W. \& Anders, S. Moderated estimation of fold change and dispersion for RNAseq data with DESeq2. Genome Biol 15, 550 (2014).

44. Peng, T., et al. A BaSiC tool for background and shading correction of optical microscopy images. Nature Communications 8, 14836 (2017).

45. Anders, S. \& Huber, W. Differential expression analysis for sequence count data. Genome Biol 11, R106 (2010).

46. Benjamini, Y., Drai, D., Elmer, G., Kafkafi, N. \& Golani, I. Controlling the false discovery rate in behavior genetics research. Behav Brain Res 125, 279-284 (2001).

47. Harrell, F.E., Jr., Lee, K.L., Califf, R.M., Pryor, D.B. \& Rosati, R.A. Regression modelling strategies for improved prognostic prediction. Stat Med 3, 143-152 (1984).

48. Grubbs, F.E. Procedures for Detecting Outlying Observations in Samples. Technometrics 11, 1-21 (1969).

49. R Core Team. R: A Language and Environment for Statistical Computing. Vienna, Austria. $R$ Foundation for Statistical Computing (2015). 


\section{Table 1}

\begin{tabular}{lc}
\hline & \\
\hline Number of subjects (\% males) & $252(58.2 \%)$ \\
\hline Age at enrolment & $65.0 \pm 0.7 \mathrm{yr}$. \\
Age of onset ( $1^{\text {st }}$ reported symptoms) & $62.6 \pm 0.7 \mathrm{yr}$. \\
Disease duration at enrolment & $28.8 \pm 2.1 \mathrm{~m}$. \\
ALSFRS-R at enrolment & $35.9 \pm 0.5$ \\
El-Escorial (Definite/probable/lab- & $67 / 102 / 26 / 38 / 2 / 17$ \\
supported/possible/suspected/other) & \\
Bulbar onset / total & $83 / 252$ \\
Riluzole treated / total & $167 / 252$ \\
ALS Family history / total & $9 / 252$ \\
C9ORF72 genetics / total & $15 / 252$ \\
\hline
\end{tabular}

573
Table 1. Summary of demographic and clinical characteristics of ALS samples used for the survival study. ALSFRS-R: ALS functional rating scale. Data are presented as mean \pm SEM. 


\begin{tabular}{lcc}
\hline & Cohort I & Cohort II \\
\hline Number of subjects (\% males) & $22(81.8 \%)$ & $26(53.8 \%)$ \\
Age at enrolment & $65.0 \pm 1.5 \mathrm{yr}$. & $63.5 \pm 2.4 \mathrm{yr}$. \\
Age of onset (1 ${ }^{\text {st }}$ reported symptoms) & $62.0 \pm 1.7 \mathrm{yr}$. & $60.4 \pm 2.4 \mathrm{yr}$. \\
Disease duration at enrolment & $37.2 \pm 8.2 \mathrm{~m}$ & $37.0 \pm 8.5 \mathrm{~m}$. \\
ALSFRS-R at enrolment & $41.0 \pm 1.2$ & $36.6 \pm 1.4$ \\
El-Escorial (Definite/probable/lab- & $4 / 7 / 6 / 5 / 0$ & $3 / 14 / 3 / 3 / 3$ \\
supported/possible/other) & & \\
Bulbar onset / total & $8 / 22$ & $6 / 26$ \\
Riluzole treated / total & $13 / 22$ & $15 / 26$ \\
ALS Family history / total & $1 / 22$ & $1 / 26$ \\
C9ORF72 genetics / total & $0 / 22$ & $3 / 26$ \\
\hline
\end{tabular}

578

579

580

581
Table 2. Summary of demographic and clinical characteristics of ALS samples used for the longitudinal study. ALSFRS-R: ALS functional rating scale. Data are presented as mean \pm SEM. 
A

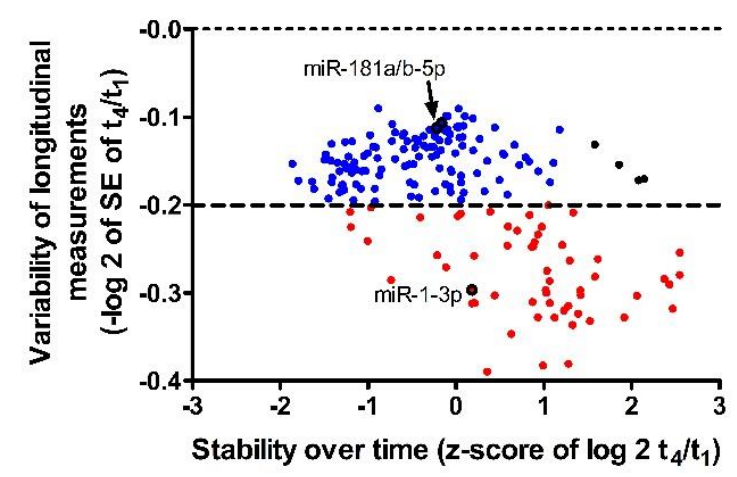

B

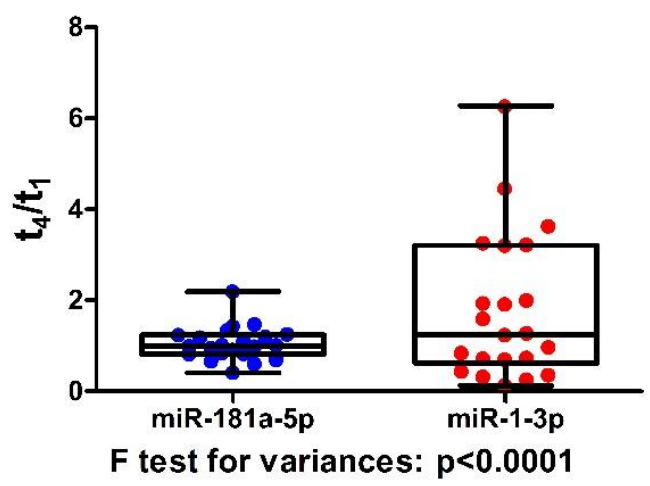

Figure 1. Assessment of plasma miRNA stability during ALS course. (A) The $x$-axis denotes the standardized change in miRNA levels between the first and last measurements (number of standard deviations (SD) for $\log 2$-transformed $\mathrm{t}_{4} / \mathrm{t}_{1}$ ratios), relative to the average change of all 187 sequenced miRNAs. The y-axis denotes the variability in measurements, per-miRNA for 187 sequenced species between the 22 individuals (- $\log 2$-transformed values of the standard error of $\mathrm{t}_{4} / \mathrm{t}_{1}$ ratios). Features above (blue) or below (red) the stability threshold set at -0.2 units. (B) Variability of miR-181a-5p and miR-1-3p between fourth and first phlebotomy (t4/t1) in 22 ALS patients. F test $\mathrm{p}<0.0001$. 


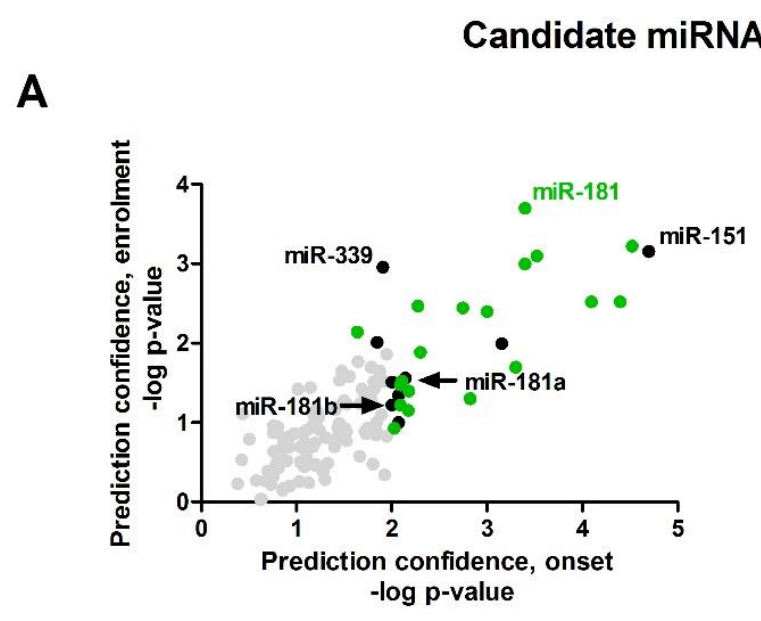

C

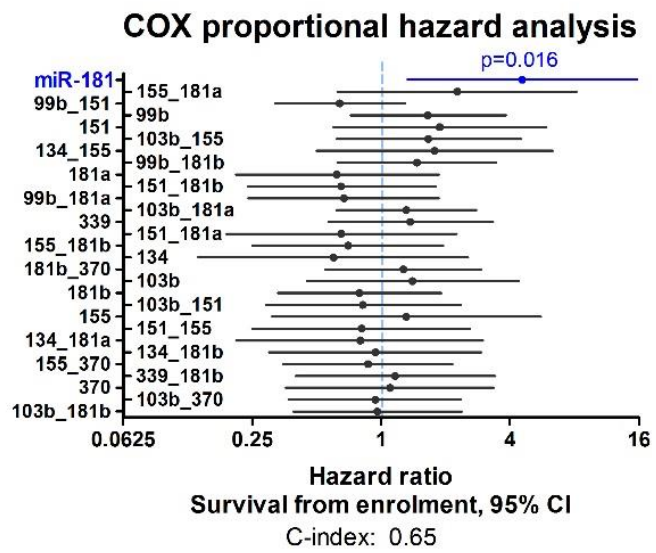

B

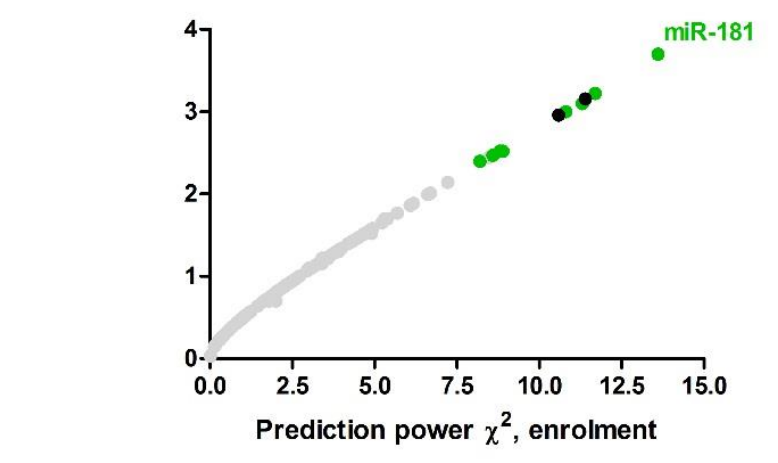

Bootstrapping biomarker
selection with backward elimination

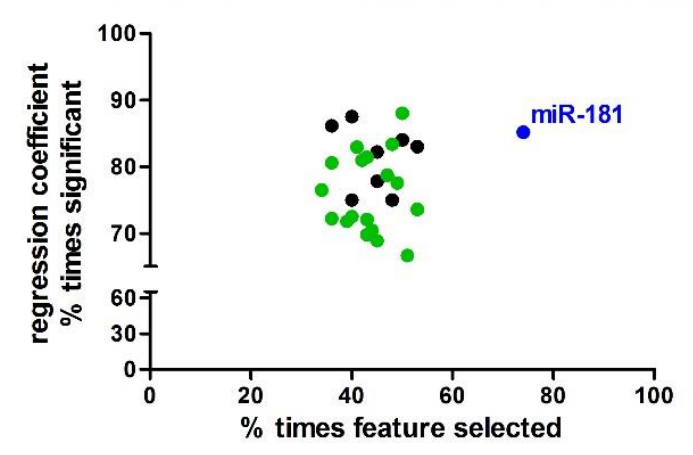

Figure 2. Identification of candidate miRNAs that predict ALS patient survival. (A) Scatter plot, assessing separation of survival by high and low (dichotomized) levels of 123 miRNA features (i.e., single miRNAs and miRNA pairs). Logrank test from study enrollment (y-axis) or first symptoms (onset, $\mathrm{x}$-axis). Log 10 transformed p-values of logrank chi^2 values. The optimal threshold was calculated per miRNA in a discovery cohort of 126 patients by Evaluate Cutpoints algorithm ${ }^{21}$. Single miRNA (black, namely, miR-103b-3p, miR-134-5p, miR-151-5p, miR-1555p, miR-181a-5p, miR-181b-5p, miR-339-3p, miR-370-3p, miR-99b-5p) or miRNA pairs (green), displaying a p-value $\leq 0.01(\log 10$ transformed values $\geq 2)$, and grey: insignificant. The paired feature composed of miR-181a-5p with miR-181b-5p is called for simplicity miR-181 throughout the manuscript. (B) Scatter plot of effect (logrank chi^2 values, $\mathrm{x}$-axis), against confidence (pvalue, y-axis). Color code as in panel A. (C) Forest plot of mortality hazard ratios calculated by multivariate Cox study from enrolment for all significant miRNAs and miRNA pairs in Figure 2A (colored black and green). Blue features - displaying a p-value $\leq 0.05$, black: insignificant by Wald test. (D) Bootstrap-based model selection according to Akaike's information criteria (AIC). Backwards feature elimination for 29 features (miRNA or miRNA pairs) passing $\mathrm{p}$-value $\leq 0.01$ filtering in the logrank test (Figure 2A). Plot of the percentage of times each feature was selected (x-axis), against the percentage of times the Cox regression coefficient of this feature was significant in repeated measurements (y-axis). 


\section{Discovery cohort $(n=126)$}

A

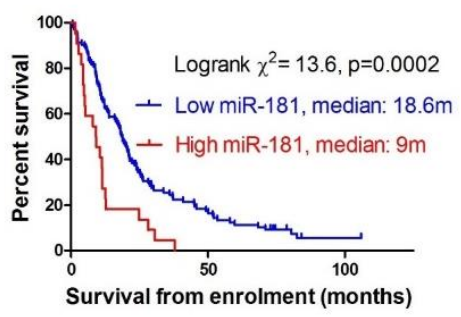

B

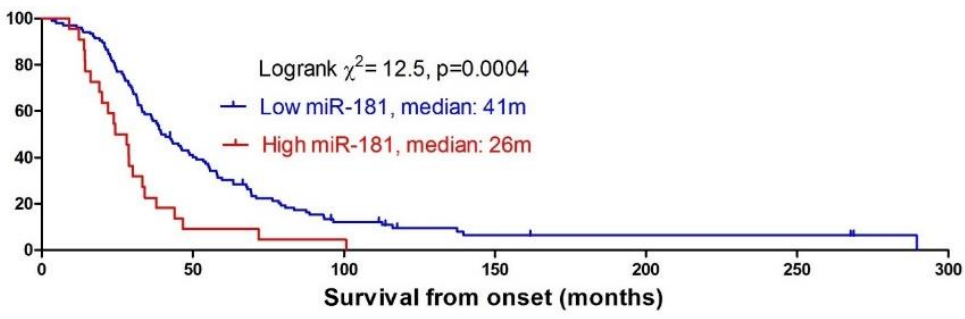

\section{Replication cohort $(n=122)$}

C

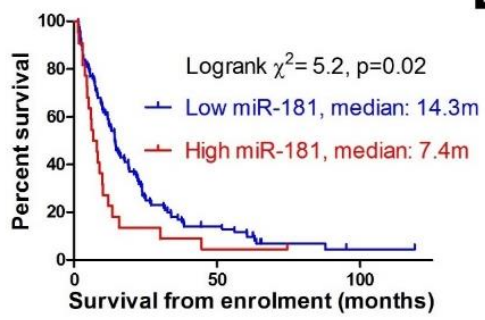

D

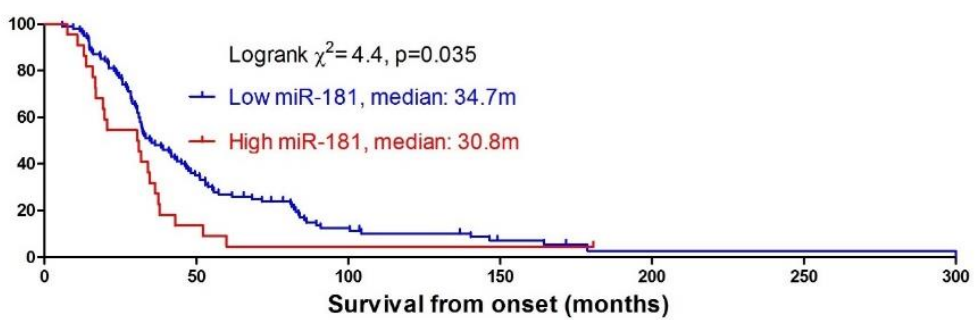

\section{Merged cohort $(n=248)$}

E

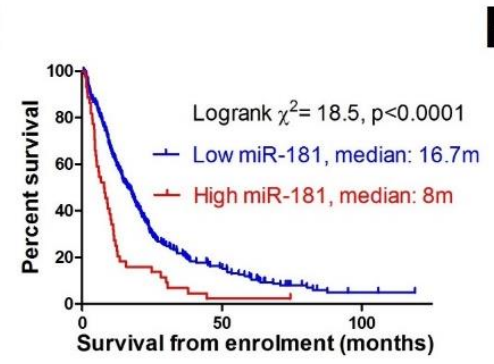

F

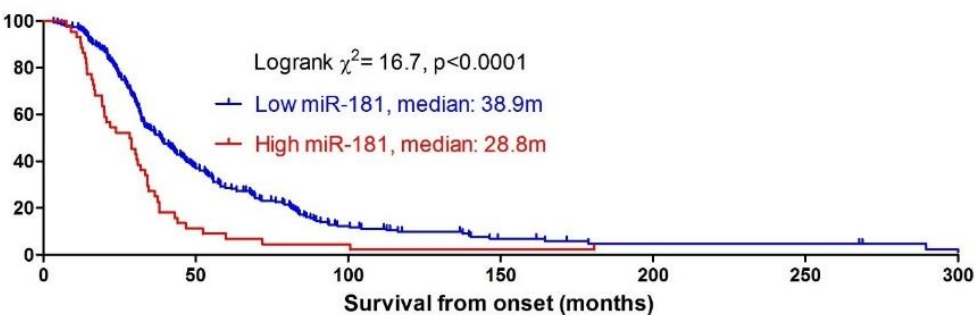

G

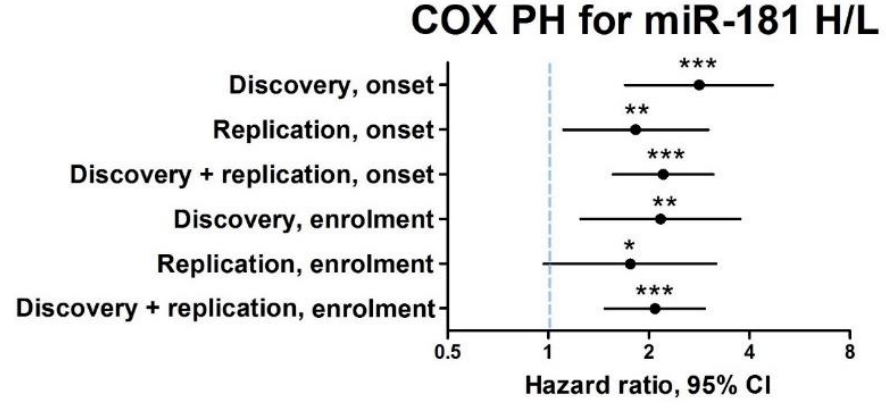


620 Figure 3. miR-181 is a prognostic biomarker of ALS. Cumulative survival (Kaplan-Meier) 621 curves for miR-181 (104 patients with subthreshold (blue) vs. 22 patients with suprathreshold (red) 622 miR-181 levels) in discovery cohort from enrolment (A) and onset (B). Kaplan-Meier curves for 623 miR-181 (100 patients with subthreshold (blue) vs. 22 patients with suprathreshold (red) miR-181 624 levels) in replication cohort from enrolment (C) and onset (D). Kaplan-Meier curves on the two 625 cohorts merged (204 patients with subthreshold (blue) vs. 44 patients with suprathreshold (red) 626 miR-181 levels) from (E) enrolment or $(\mathbf{F})$ onset. Forest plot showing results of multivariate Cox 627 proportional hazard analysis of miR- 181 corresponding to KM curves in panels A-F $(\mathbf{G}) .{ }^{*} \mathrm{p}<0.05$, 628 one-tailed Wald test. ${ }^{*} \mathrm{p}<0.01$, one or two-tailed Wald test. ${ }^{*} * \mathrm{p}<0.001$, two-tailed Wald test. 629 Mean $\pm 95 \%$ CIs. 
A

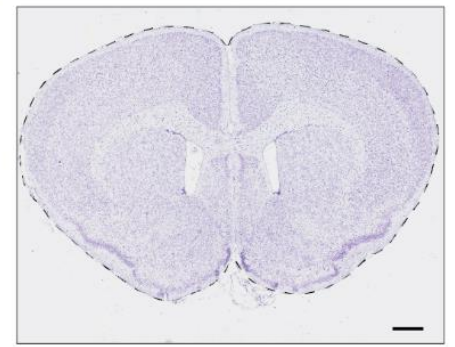

B

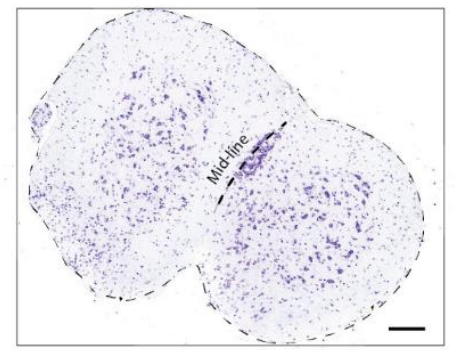

C

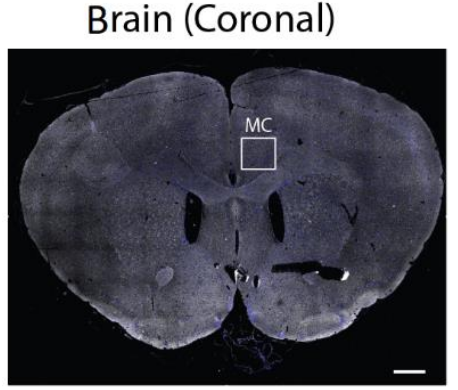

D Spinal cord (Transverse)

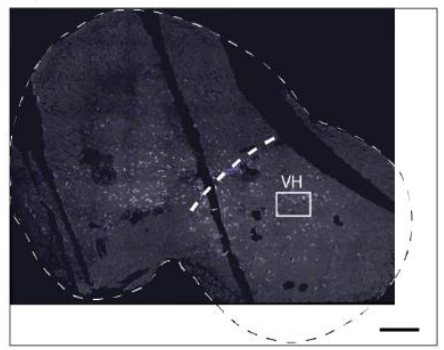

E

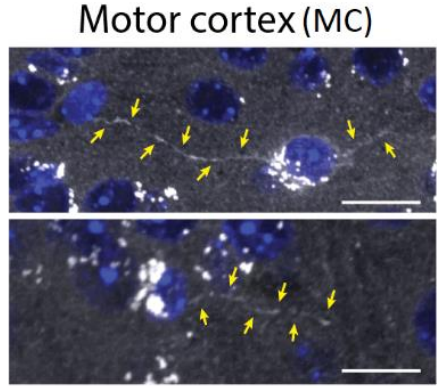

$\mathbf{F}$

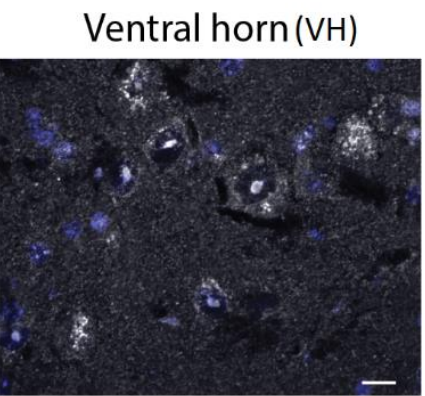

632 Figure 4. miR-181a-5p localizes to neuronal soma and neurites in mouse brain and spinal

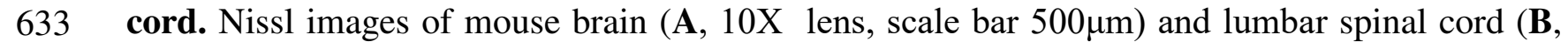
634 magnification 10X, scale bar $200 \mu \mathrm{m}$ ), and corresponding brain (C) and ventral horn (D) miR635 181a-5p in situ hybridization micrographs. miR-181 is detected in critical motor neuron soma and 636 neurites (E, motor cortex, $63 \mathrm{X}$ lens, scale bar $15 \mu \mathrm{m}$ ), and in motor neurons of the spinal cord 637

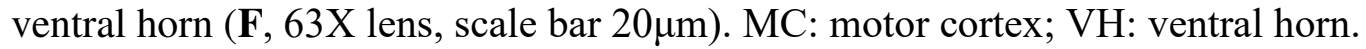


COX PH for miR-181, NfL and NfL + miR181

A

B

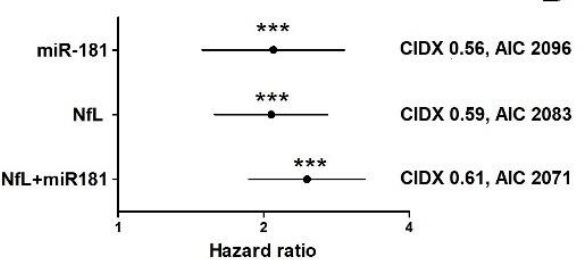

Survival from enrolment, $95 \% \mathrm{Cl}$

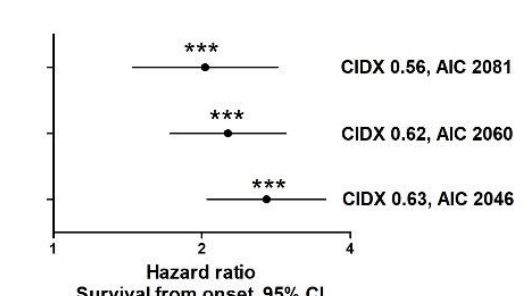

KM curves for NfL only

C

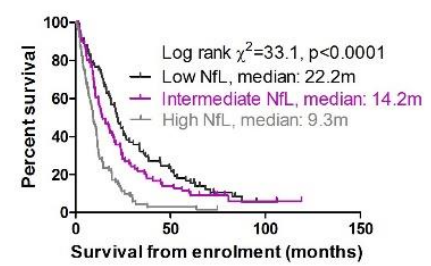

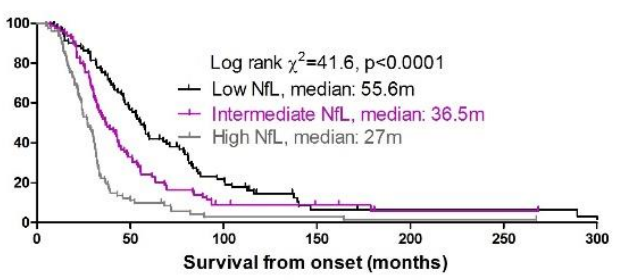

KM curves for NfL with miR-181 in intermediate range

E

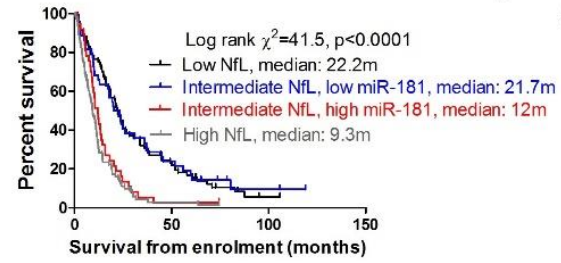

COX PH for miR-181 in NfL tertiles

G

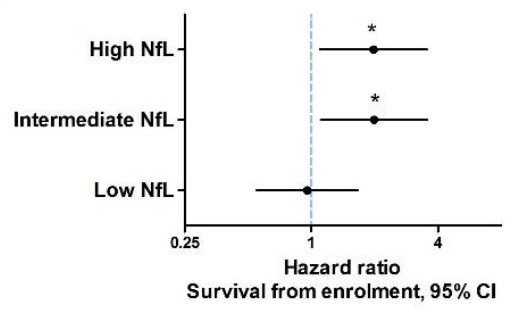

H

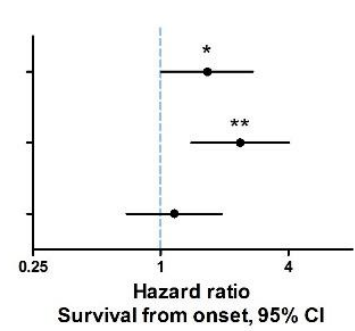

Predicted vs observed survival

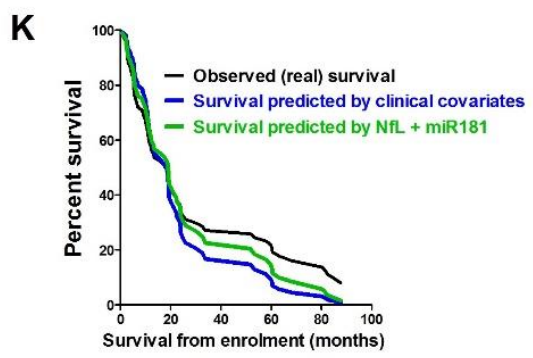

COX PH for NfL + miR181 and eight clinical covariates

I

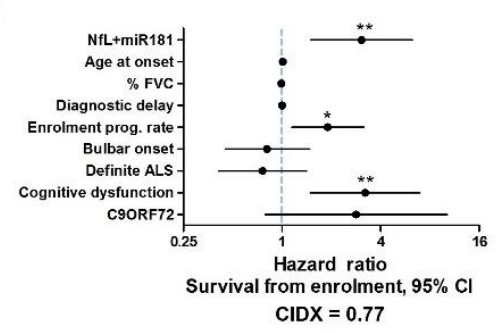

J

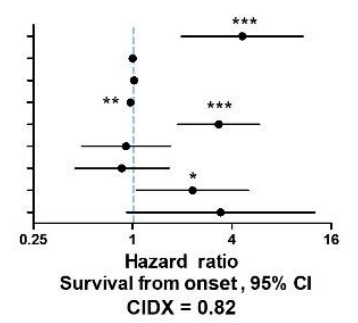


642 Figure 5. Superior accuracy for combination of miRNA and NfL biomarkers in prognosis

643 analysis. Cox proportional hazard analysis for miR-181, NfL and a combinatorial predictor

$644 \mathrm{NfL}+\mathrm{miR} 181$ in 243 patients with both miR-181 and NfL measurements (threshold values: miR-

64518171,000 UMIs, NfL $82.2 \mathrm{pg} / \mathrm{ml}$, NfL+miR181 upper 118 vs lower 125), from enrolment (A)

646 or onset (B). CIDX- concordance index. AIC -Akaike's information criteria. Kaplan Meier

647 curves calculated from enrolment (C) or onset (D) based on tertile stratification of plasma NfL

648 levels. (NfL threshold values by Simoa assay: < $59 \mathrm{pg} / \mathrm{ml}$ (low) ;59 $-109.8 \mathrm{pg} / \mathrm{ml}$ (intermediate);

$649>109.8 \mathrm{pg} / \mathrm{ml}$ (high). Kaplan Meier curves calculated from enrolment (E) or onset (F), whereby

650 the middle tertile of samples with intermediate NfL levels is further subdivided by miR-181

651 levels. Forest plots of mortality hazard ratio, calculated by survival length from enrolment (G) or

652 onset $(\mathbf{H})$ for high vs low miR-181 levels in the three NfL tertiles. Forest plots of mortality

653 hazard ratio, calculated by survival length from enrolment (I) or onset (J) for combined miRNA-

654 protein predictor, $\mathrm{NfL}+\mathrm{miR} 181$ and 8 clinical covariates ${ }^{23}$ on a subset of 75 patients. Mean \pm

$65595 \%$ CI, Wald test $* \mathrm{p}<0.05, * * \mathrm{p}<0.01, * * * \mathrm{p}<0.001$. (K) Observed survival curve (black) vs.

656 prediction based on NfL + miR181 (green) or by 8 clinical covariates (blue), in a subset of 75

657 patients. 
660

A

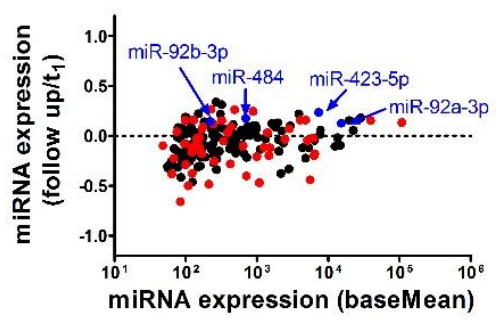

B

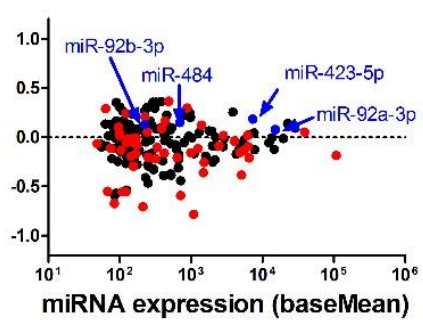

C

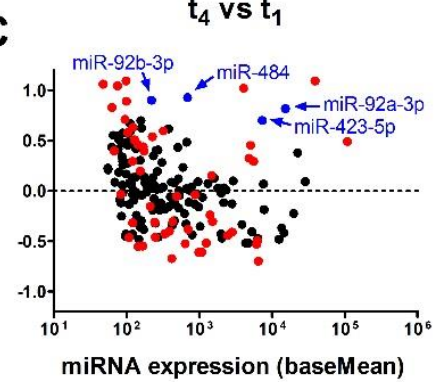

661

662

663

664

665
Figure S1. (A-C) MA plots of differential miRNA expression upon repeated sampling relative to the first phlebotomy. Red features denote miRNAs with statistically significant change in levels. 
A

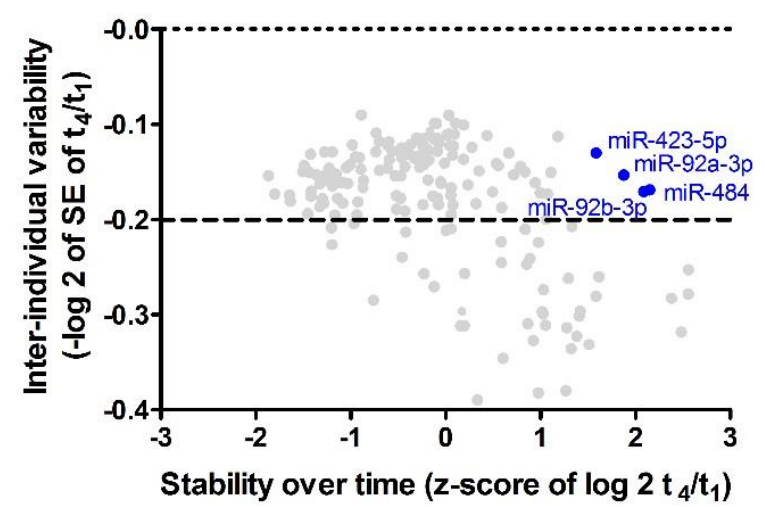

B
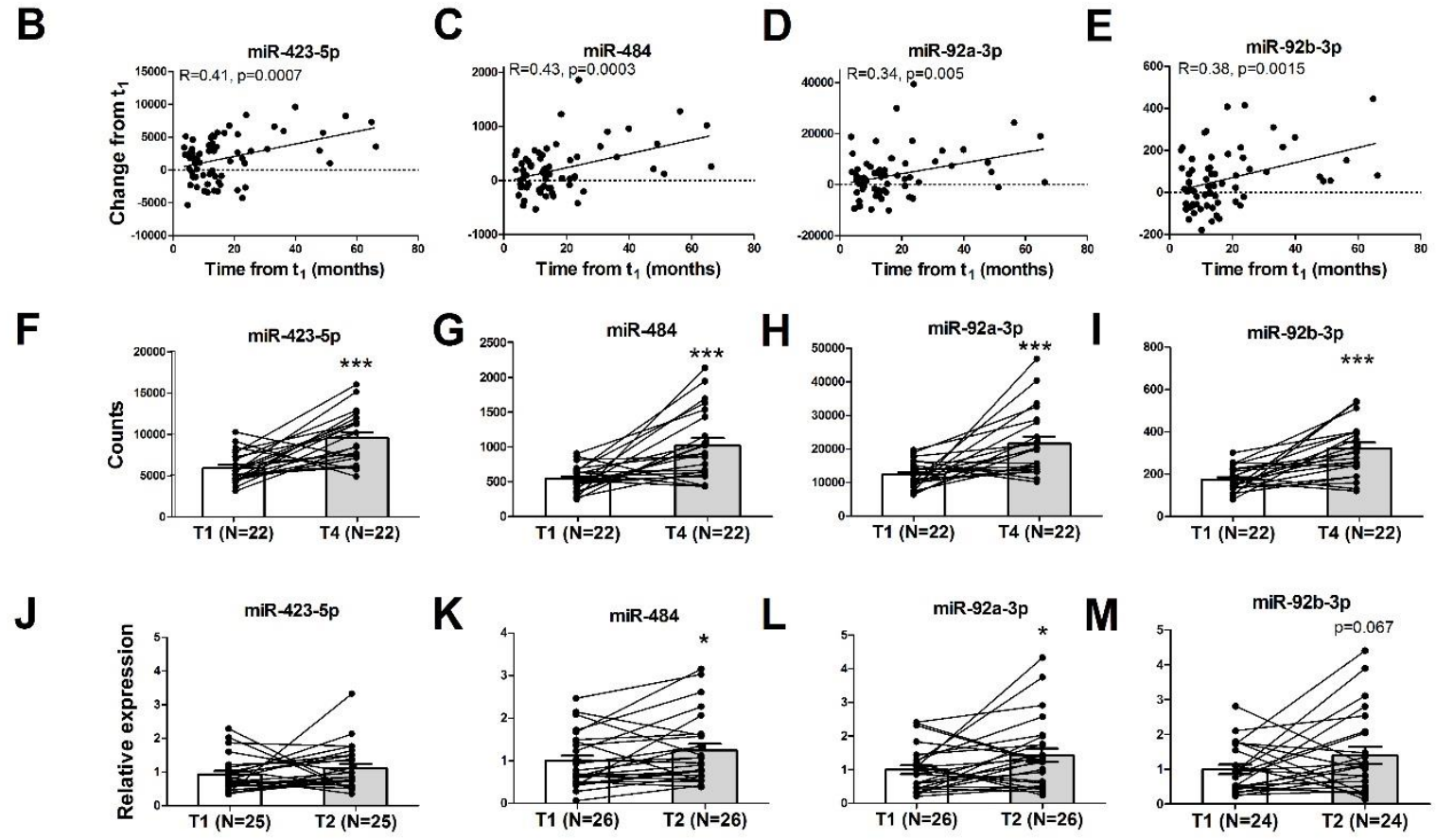

Figure S2. Analysis of miRNAs that increase during ALS course. inter-individual variability, but increased with the disease course, suggesting that, although they are not suited for prognostic use, they could potentially monitor disease progression. (miR423/484/92a/b, $\mathrm{t}_{4} / \mathrm{t}_{1}>1.5 \mathrm{SD}, \mathrm{X}$-axis) (A). Temporal changes in the levels of (B) miR-423-5p, (C) miR-484, (D) miR-92a-3p, or (E) miR-92b-3p and revealed correlation with time passing from enrolment (in months). Spaghetti plots of individual patient trajectories $\left(\mathrm{t}_{1-1} \mathrm{t}_{4}\right)$ denoted for $(\mathbf{F}) \mathrm{miR}$ 423-5p, (G) miR-484, (H) miR-92a-3p, or (I) miR-92b-3p. Time intervals: $\mathrm{t}_{1}-\mathrm{t}_{2} 6.3 \pm 0.3 \mathrm{~m}$; $\mathrm{t}_{1}-\mathrm{t}_{3}$ $13.0 \pm 0.3 \mathrm{~m} . ; \mathrm{t}_{1}-\mathrm{t}_{4} 32.7 \pm 3 \mathrm{~m}$. Disease duration: $\mathrm{t}_{1} 28.8 \pm 3 \mathrm{~m}$.; $\mathrm{t}_{4} 61.5 \pm 3 \mathrm{~m}$. Validation of changes to miRNA levels in an independent replication cohort ( $N=26$ individuals, Table 2). Spaghetti plots of individual patient trajectories $\left(\mathrm{t}_{1}-\mathrm{t}_{2} 13.7 \pm 1.6\right.$ months) in a replication cohort, for $(\mathbf{J}) \mathbf{m i R}-423$ 5p, p=0.17 (K) miR-484, p=0.02; (L) miR-92a-3p, p=0.02; or (M) miR-92b-3p, p=0.067. Onetailed t-test. Together, miR-484 and miR-92a/b may be considered as candidate molecular 
682 biomarkers of functional decline over the course of disease. Mean \pm SEM. $* p<0.05, * * * p<0.001$, 683 one-tailed paired t-test or Wald test. Analysis of a single miR-423-5p sample and two miR-92b$6843 p$ samples in the replication cohort deviated from the mean according to Grubbs test and these 685 were excluded as outliers.

686

687

688

689

690

691

692

693

694

695

696

697

698

699

700

701

702

703

704

705

706

707

708

709

710 
A

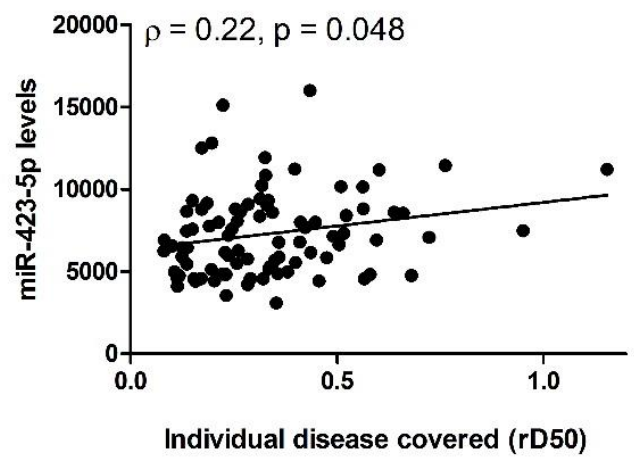

C

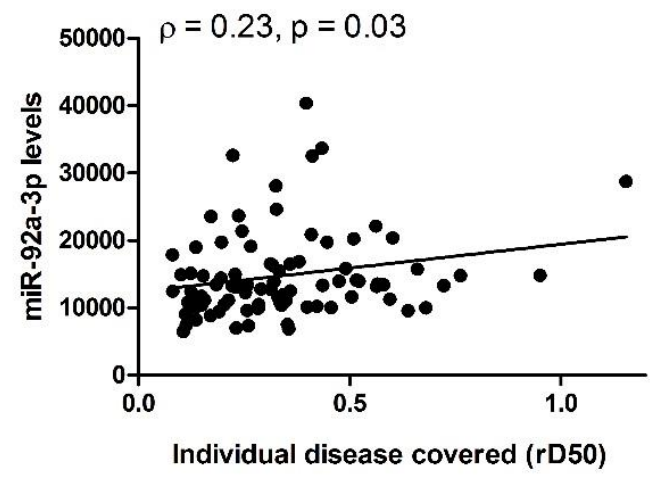

B

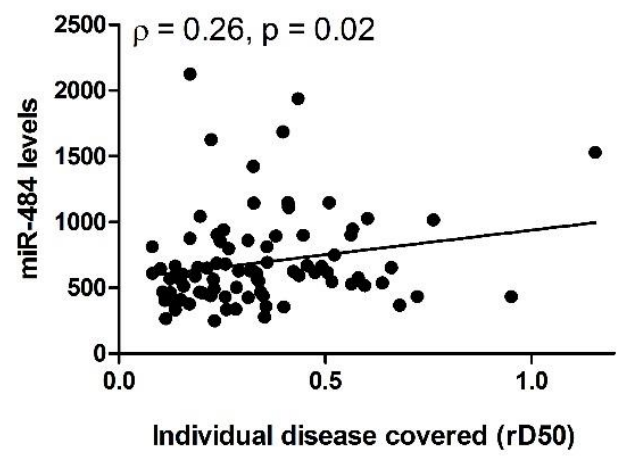

D

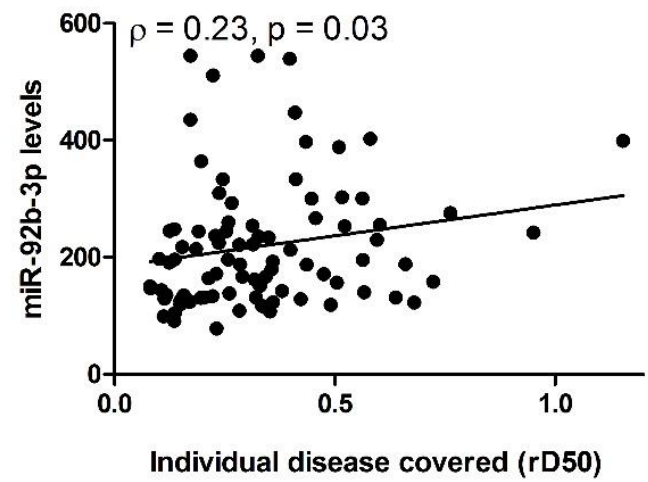

711

712

713

714

715

716

717

718

719

720

721

722

723

724

725

726

727

728

729

730

731

732

733

Figure S3. Longitudinal change of miRNA-423-5p, miR-484, miR-92a and miR-92b with disease progression. Correlation between the relative disease covered (rD50) in longitudinal plasma collections (X-axis) and levels of (A) miR-423-5p, (B) miR-484, (C) miR-92a-3p, and (D) miR-92b-3p. The relative D50 (rD50) is a derivative of ALS Functional Rating Scale-Revised (ALSFRS-R) decay that reveals the disease covered by individual patients independent of the rate of progression ${ }^{24,40}$. For example, an rD50 of 0.0 signifies ALS onset, and 0.5 signifies the timepoint where functionality is reduced by half. Longitudinal miR-484/92a/b levels in blood correlated with rD50 at the time of sampling (Figure S3A-D). 
A

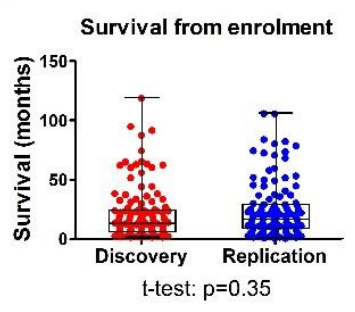

E

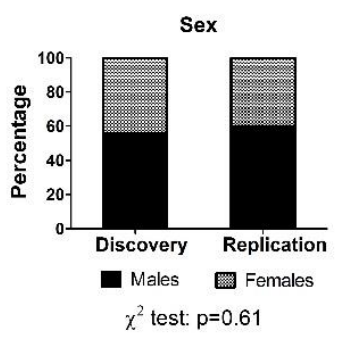

B

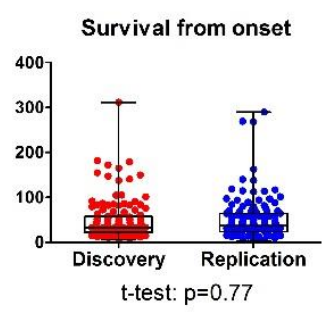

$\mathbf{F}$

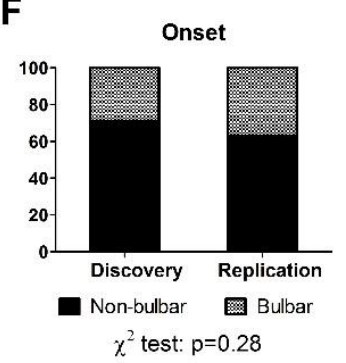

C

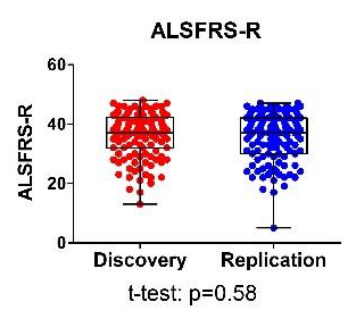

G

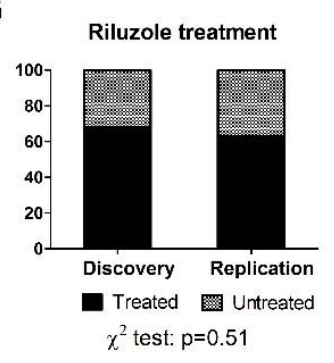

D

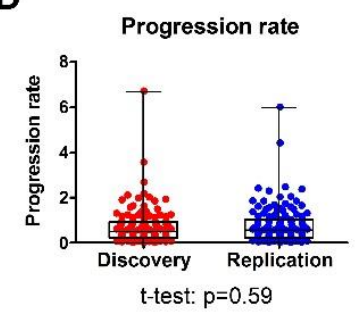

H

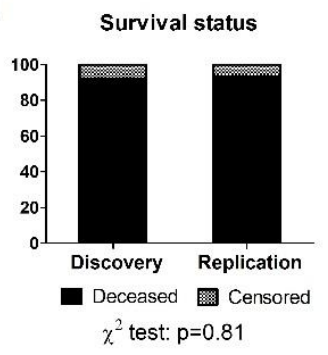

\section{$\mathrm{N}=126$ for both cohorts}

Figure S4. Clinical features are comparable between cohort I and cohort II. (A) Survival from enrolment (B) survival from symptom onset (C) ALSFRS-R score at enrolment (D) progression rate at enrolment (E) sex distribution (F) onset site distribution (G) Riluzole treatment status $(\mathbf{H})$ number of censored patients. 

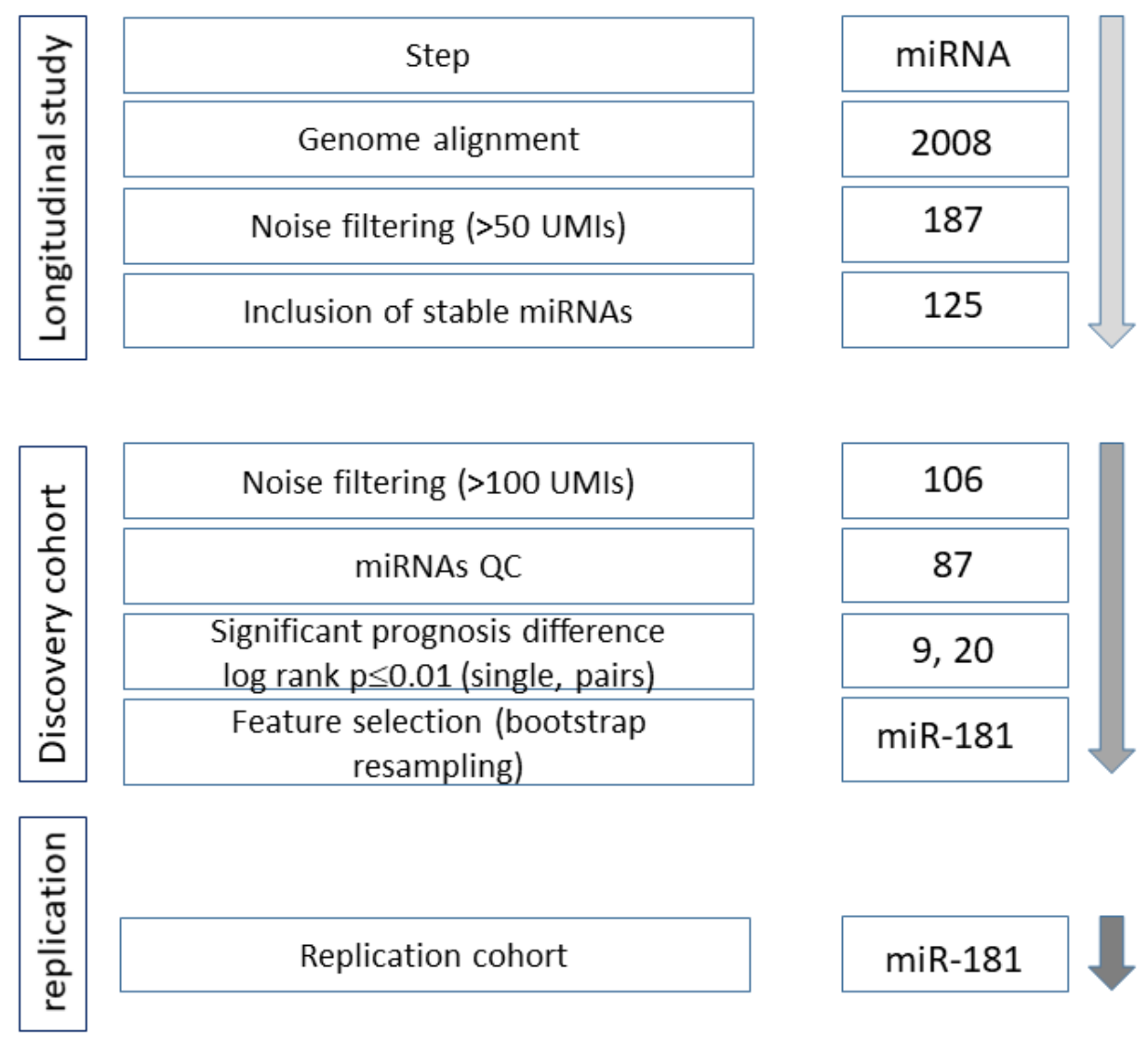

Figure S5. Pipeline for selecting miRNAs as candidate prognostic markers. 2008 miRNAs were aligned to the genome in the longitudinal study and out of them, 187 miRNAs which exhibited >50 UMI counts in 60\% of the samples were included in further analysis. 125 out of the 187 miRNAs were longitudinally stable with low interindividual variability (green features in Figure 1A). In the discovery cohort, 106 out of these 125 miRNAs passed a filtering criterion of average UMI counts $>100$ across all samples, and were analyzed for prognosis differences between low and high level in the discovery cohort. 19 miRNAs were further excluded after additional QC based on logrank analysis (opposite directions of prognosis differences between members of the same miRNA family, e.g. miR-27a and miR-27b), and the remaining 87 miRNAs were assessed for logrank and $p$ values for prognosis differences as demonstrated in Figure 2. 9 out of these 87 miRNAs displayed logrank $p \leq 0.01$, and all of their possible pairs $(9 * 8 / 2=36)$, derived from multiplication of the levels of two single miRNAs, were further assessed for prognosis differences. Nine single miRNAs and 20 miRNA pairs which displayed logrank $\mathrm{p} \leq 0.01$ were further subjected to feature selection by bootstrap resampling, whereby features had to be selected $>70 \%$ of the bootstrap samples and display statistical significance in $>85 \%$ of the samples in which it selected, in order to be tested as a prognostic marker on the full discovery cohort. miR-181 was the only feature fulfilling those criteria, hence it was tested in the discovery cohort, and exhibited significant survival differences and hazard ratios, both in the discovery cohort and when validated 


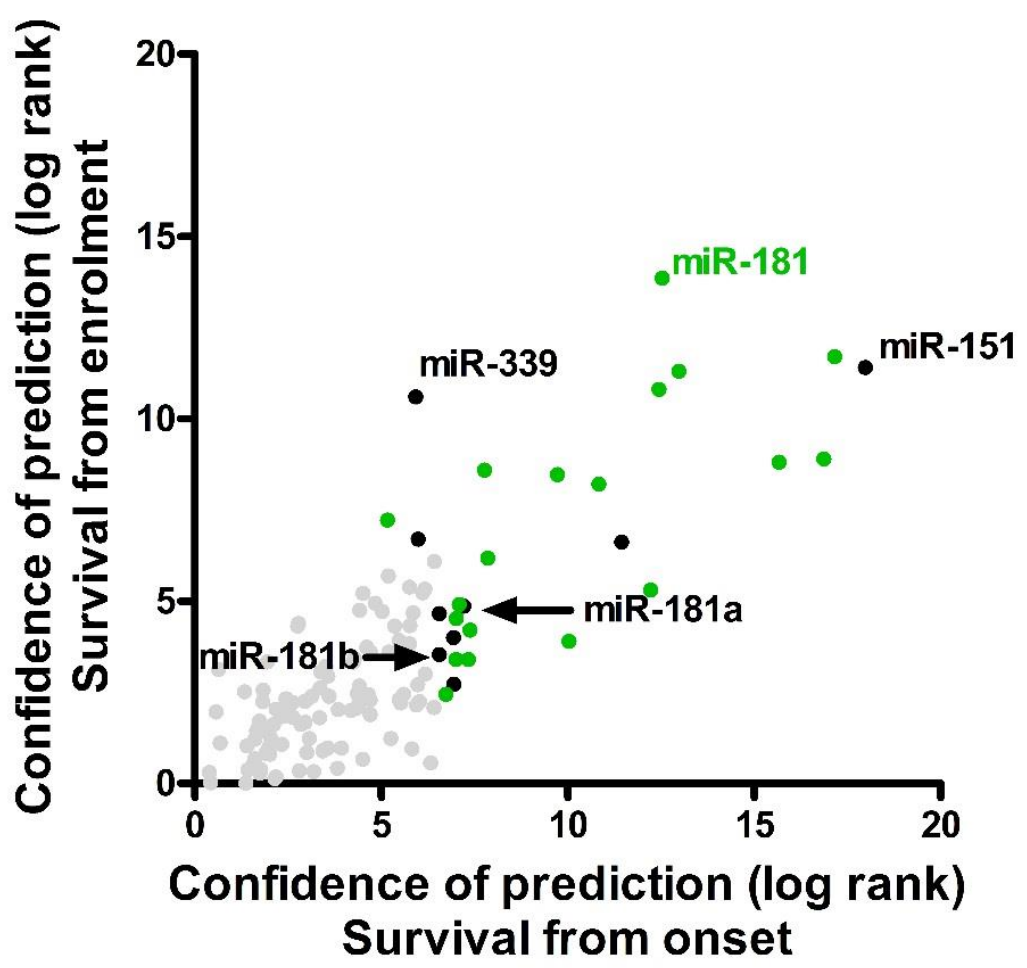

Figure S6. Scatter plot, assessing agreement between separation of survival by 123 miRNA features, by logrank test from study enrollment ( $\mathrm{Chi}^{\wedge} 2$, y-axis) or first symptoms (onset, $\mathrm{Chi}^{\wedge} 2$, $\mathrm{x}^{-}$ axis). The optimal threshold was calculated per miRNA in a discovery cohort of 126 patients by ${ }^{21}$. Single miRNA (black) or miRNA pairs (green), displaying a p-value $\leq 0.01$ (log 10 transformed values $\geq 2$ ), and grey: insignificant. 
A

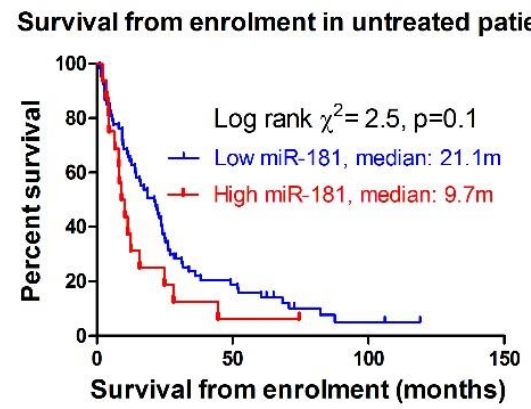

C Survival from enrolment in treated patients

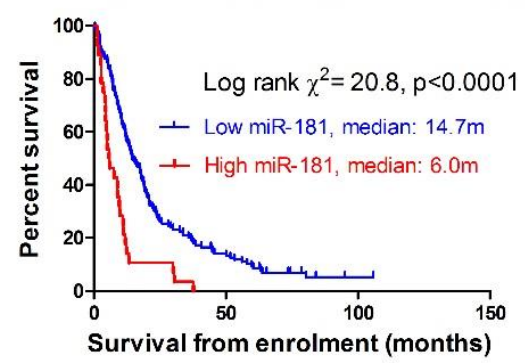

B

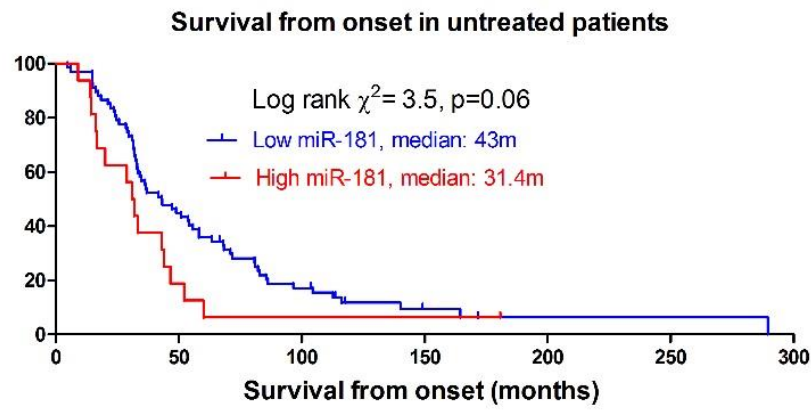

D

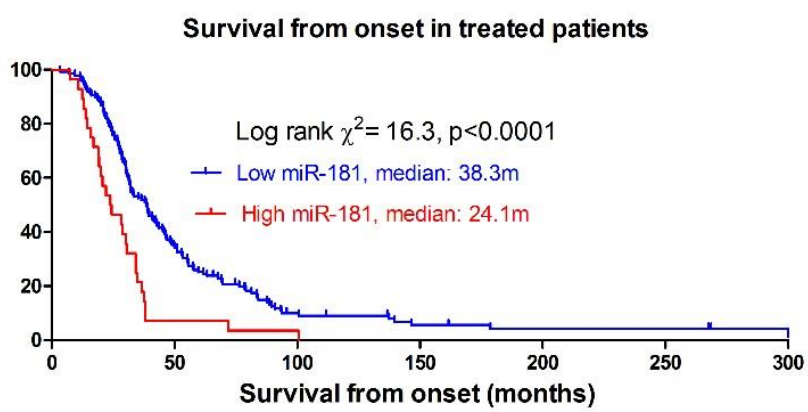

Figure S7. miR-181 levels are predictive of survival length in both untreated patients, from enrolment (A) or onset (B), and in treated patients, from enrolment (C) or onset (D). 


\section{miR-181 levels in high vs low bin}

A

miR-181 levels in discovery cohort

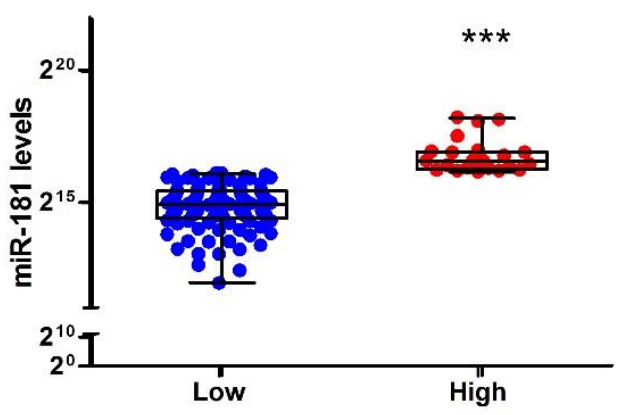

B

miR-181 levels in replication cohort

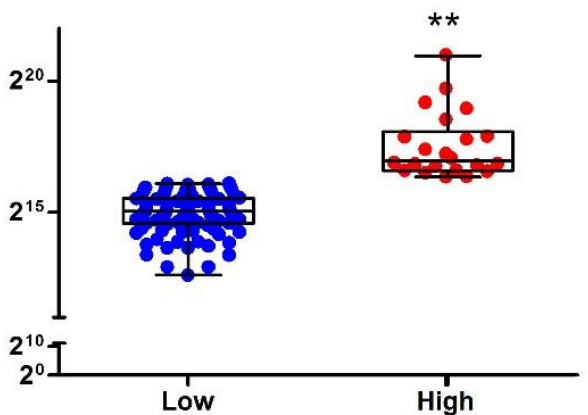

miR-181 correlation with survival, merged data

C

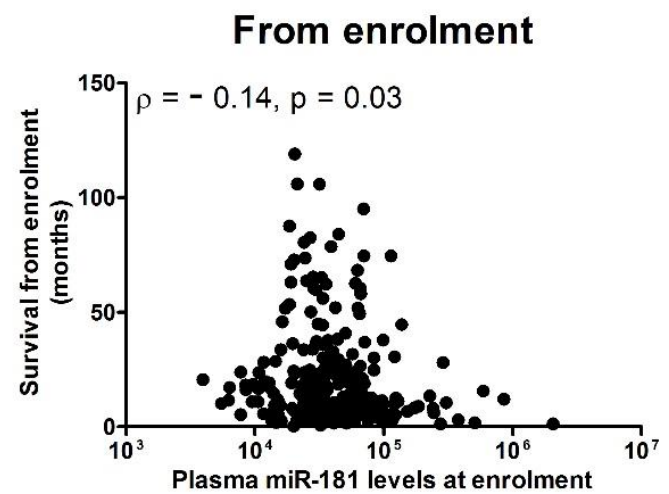

D

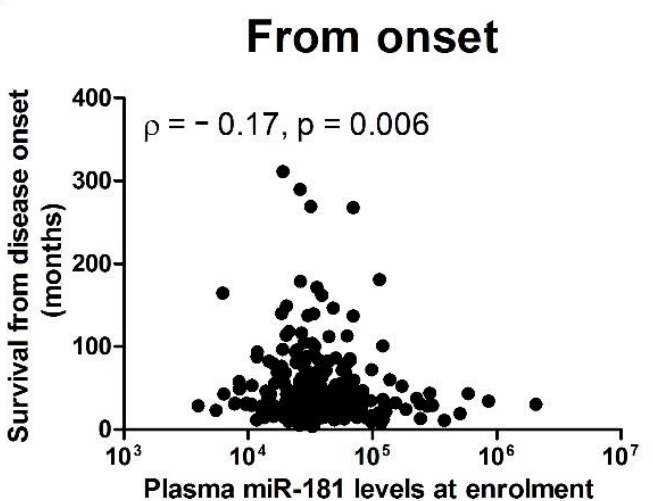

803

804

805

806

807

808

809

810

811

812

813

814

815

816
Figure S8. miR-181 levels in the high vs low expression bin, in the (A) discovery cohort and (B) replication cohort. Plots depicting inverse correlation between miR-181 levels and survival from first phlebotomy (C), or from disease onset (D). **p $<0.01$, ***p $<0.001$, t-test with Welch's correction. 
A

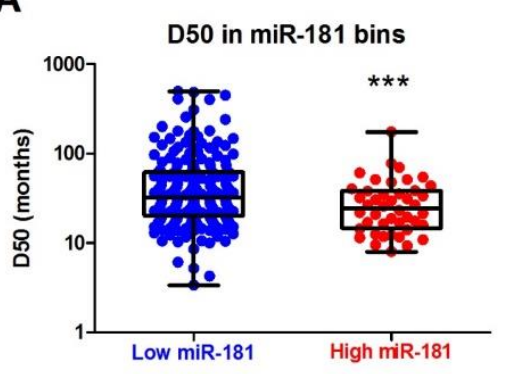

C

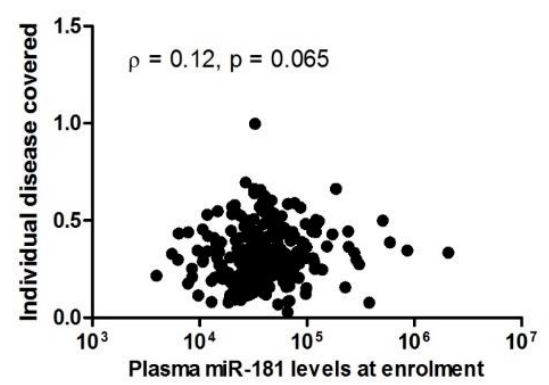

B

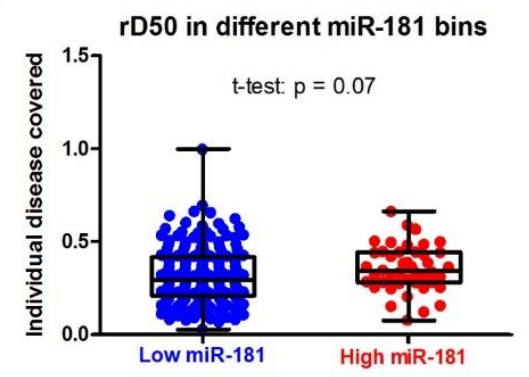

D

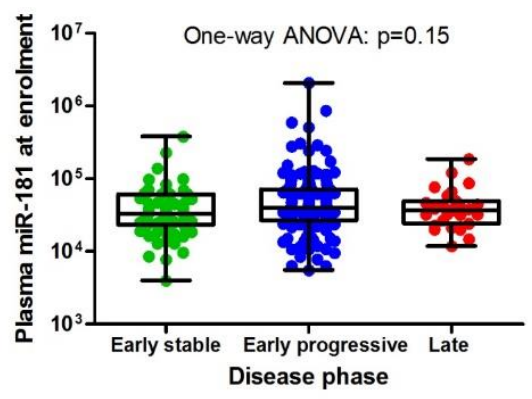

Figure S9. miR-181 levels with respect to parameters of the D50 model. (A) D50, a measure of disease aggressiveness, is significantly lower in high vs low miR-181 levels, indicating a more aggressive disease. (B) Individual disease covered, reflected by rD50 values, is not different between low and high miR-181 expression bins. (C) No correlation of miR-181 levels with individual disease covered. (D) miR-181 levels are not different between different phases of disease defined by rD50 values. ***p<0.001, t-test with Welch's correction. 
A

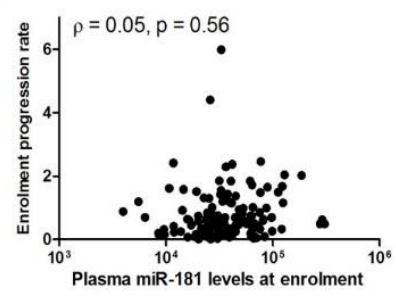

D

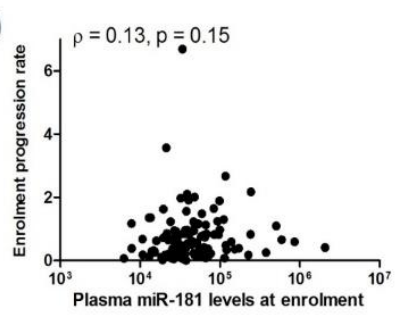

G

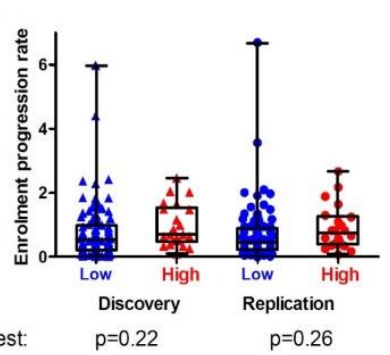

B

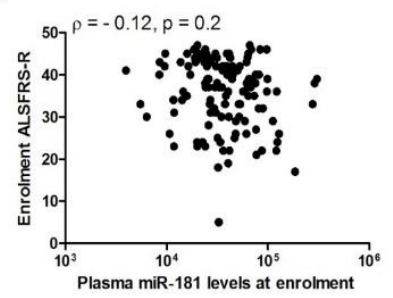

Replication $(n=122)$

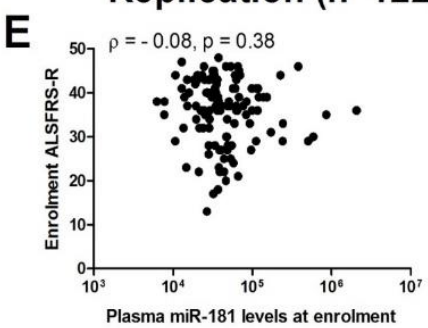

H

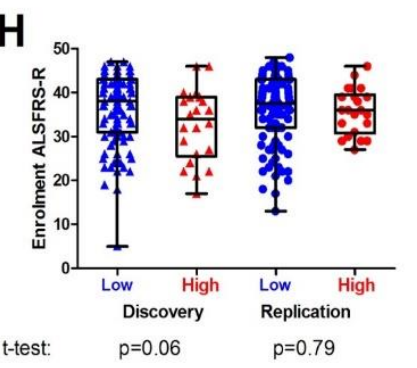

C

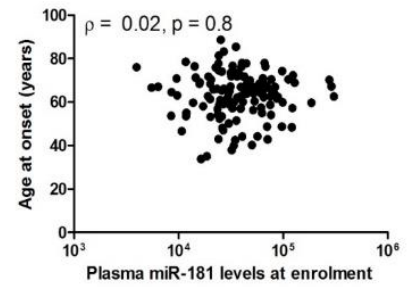

$F$

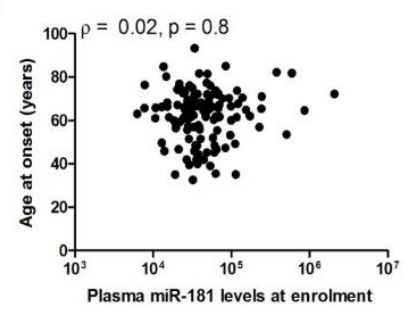

I

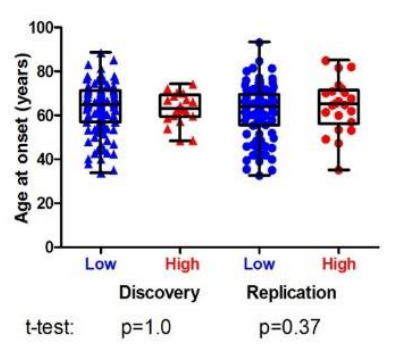

841

842

843

844

845

846

847

848

849

850

851

852

853

854

855

856

857

858

859

860

861

862
Figure S10. miR-181 levels at enrolment are not correlated with phenotypic properties in the discovery cohort (A-C), or in the replication cohort (D-F). These properties were not different between low and high miR-181 bins (G-I). 
A

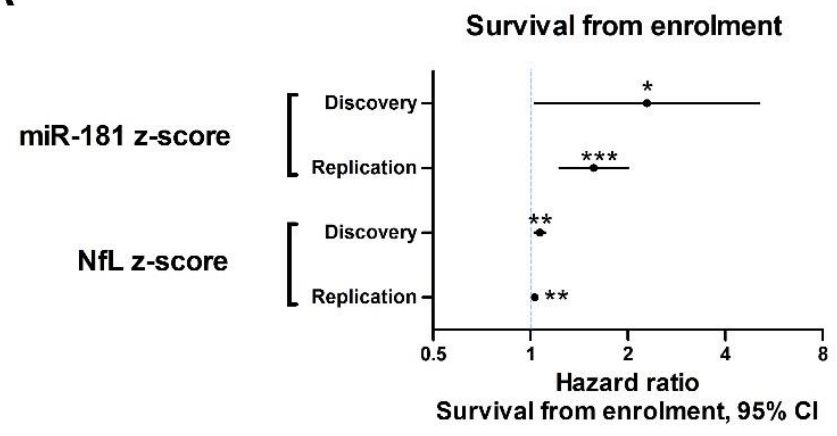

C

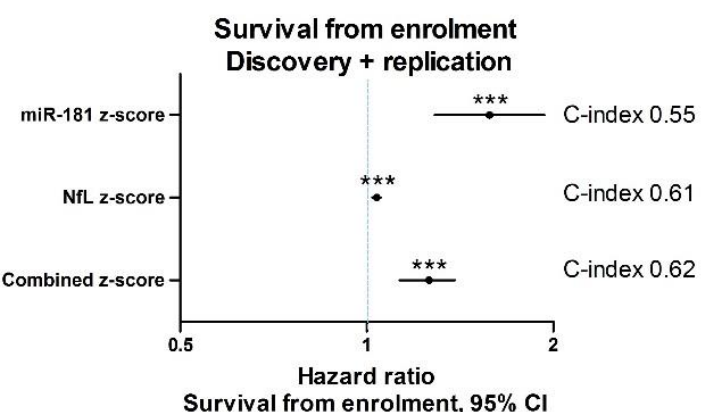

B

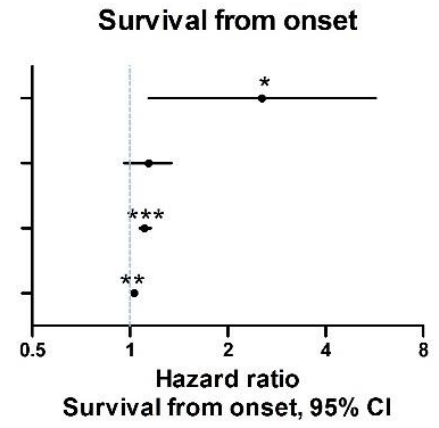

D

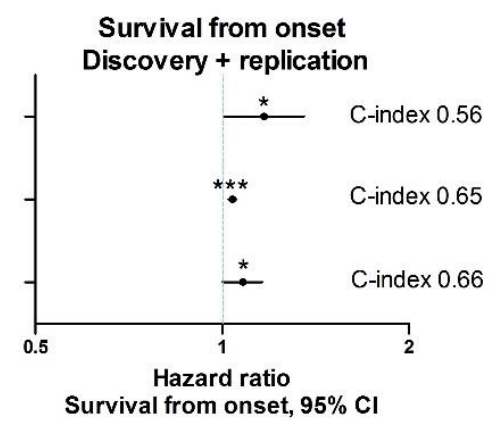

863

864

865

866

867

868

869

870

871

872

873

874

875

876

877

878

879

880

881

882

883

884

885

886

887

888

Figure S11. Multivariate Cox proportional hazard analysis for z-scores of miR-181 and NfL from enrolment (A) or onset (B) on the discovery and replication cohorts. Univariate Cox on the merged cohort (discovery + replication) for the z-scores of miR-181, NfL and the sum of the z-scores of both, from enrolment (C) or onset (D). *p $<0.05, * * p<0.01, * * * p<0.001$, Wald test. 
A

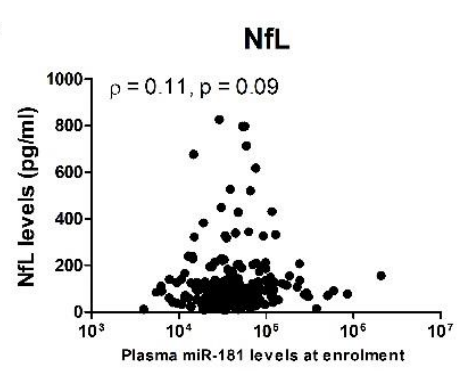

B

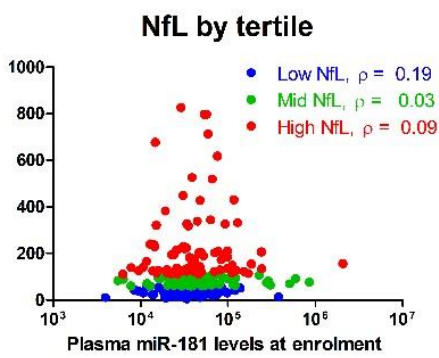

C

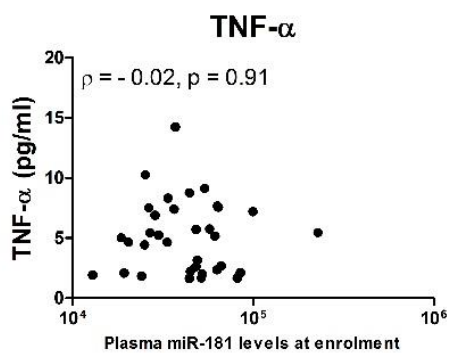

D

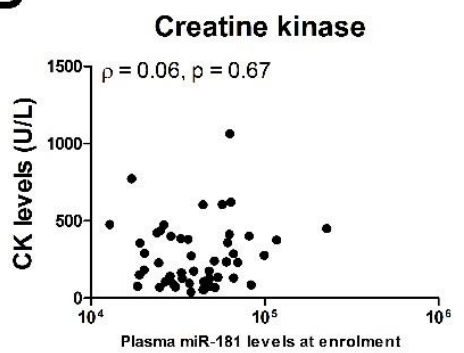

E

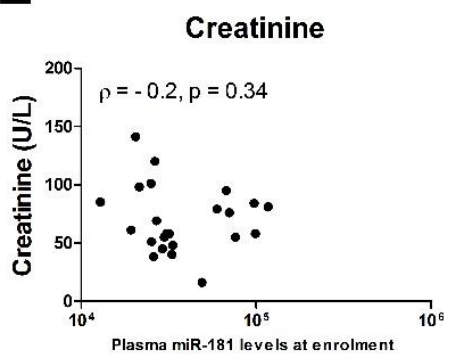

890
Figure S12. Association of miR-181 with other markers. miR-181 is not correlated with NfL levels, either in the full cohort (A) or when NfL is broken into tertiles (B). (C-E) miR-181 is not correlated with markers of muscle integrity (CK and creatinine) or inflammatory marker (TNFalpha). 


\section{Supplementary Files}

This is a list of supplementary files associated with this preprint. Click to download.

- Tables1.xls

- Tables2.xls

- Tables3.xls

- STARDChecklist.doc

- nrreportingsummary.pdf

- nreditorialpolicychecklist.docx.pdf 